\title{
Article
}

Subscriber access provided by MINCyT

\section{Plantwide control design based on the control allocation approach}

\author{
Patricio A. Luppi, Braccia Lautaro, Pablo G. Rullo, and David Alejandro R. Zumoffen
}

Ind. Eng. Chem. Res., Just Accepted Manuscript • DOI: 10.1021/acs.iecr.7b02966 • Publication Date (Web): 07 Dec 2017

Downloaded from http://pubs.acs.org on December 12, 2017

\section{Just Accepted}

"Just Accepted" manuscripts have been peer-reviewed and accepted for publication. They are posted online prior to technical editing, formatting for publication and author proofing. The American Chemical Society provides "Just Accepted" as a free service to the research community to expedite the dissemination of scientific material as soon as possible after acceptance. "Just Accepted" manuscripts appear in full in PDF format accompanied by an HTML abstract. "Just Accepted" manuscripts have been fully peer reviewed, but should not be considered the official version of record. They are accessible to all readers and citable by the Digital Object Identifier (DOI®). "Just Accepted" is an optional service offered to authors. Therefore, the "Just Accepted" Web site may not include all articles that will be published in the journal. After a manuscript is technically edited and formatted, it will be removed from the "Just Accepted" Web site and published as an ASAP article. Note that technical editing may introduce minor changes to the manuscript text and/or graphics which could affect content, and all legal disclaimers and ethical guidelines that apply to the journal pertain. ACS cannot be held responsible for errors or consequences arising from the use of information contained in these "Just Accepted" manuscripts. 


\title{
Plantwide control design based on the control
}

\section{allocation approach}

\author{
P. A. Luppi, ${ }^{* \dagger, \dagger}$ L. Braccia, ${ }^{\dagger}$ P. G. Rullo, ${ }^{\dagger}$, and D. A. R. Zumoffen ${ }^{\dagger}, \boldsymbol{\Phi}$ \\ Grupo de Ingeniería de Sistemas de Procesos (GISP), Centro Franco-Argentino de \\ Ciencias de la Información y de Sistemas (CIFASIS), CONICET-UNR, 27 de Febrero 210 \\ bis, (S2000EZP) Rosario, Argentina. \\ E-mail: luppi@cifasis-conicet.gov.ar
}

\begin{abstract}
In this work the control allocation (CA) philosophy is considered in order to propose a plantwide control (PWC) design methodology. For medium/large-scale processes where the number of actuators is greater than the number of controlled variables, a CA-based strategy can be used to distribute the total control effort among the actuators, with some attractive features. First, the CA module can explicitly handle (i) constraints on inputs (typically actuator position and rate limits), and (ii) additional control objectives (e.g. to penalize the actuators control energy). Second, CA has the potential to provide (actuator) fault tolerance without considering a control structure reconfiguration. In this paper, a decentralized control structure is proposed for computing the total control effort, which is implemented with conventional single-input
\end{abstract}

\footnotetext{
*To whom correspondence should be addressed

†Grupo de Ingeniería de Sistemas de Procesos (GISP), Centro Franco-Argentino de Ciencias de la Información y de Sistemas (CIFASIS), CONICET-UNR, 27 de Febrero 210 bis, (S2000EZP) Rosario, Argentina.

${ }^{\ddagger}$ Departamento Control, EIE, FCEIA, Universidad Nacional de Rosario, Pellegrini 250 (S2000BTP), Rosario, Argentina.

`Universidad Tecnológica Nacional - FRRo, Zeballos 1341 (S2000BQA), Rosario, Argentina. 
single-output PID controllers. In addition, a CA module is configured and two alternative algorithms are compared, namely: (i) generalized inverse plus simple saturation, and (ii) active set method for a weighted least-squares formulation. The complete structure can be designed based on steady-state process information. The well-known Tennessee Eastman case study is considered to evaluate the proposal. Several closedloop simulations are presented to show the dynamic performance of the PWC structure and the timing properties of the CA algorithm.

\section{Introduction}

Plantwide control (PWC) consists on the design and implementation of complete control strategies in order to guarantee a safe and profitable operation of (industrial) processes. To this end, several main tasks must be resolved which include the selection of (i) the process outputs to be controlled, (ii) the process inputs (actuators) to be manipulated, (iii) the control policy, which can be decentralized, centralized or a mixed strategy, (iv) the control configuration, i.e. the input-output pairings, (v) the controller technology and the corresponding tuning parameters. It is clear that for medium/large-scale processes with (substantial) interaction between units, the PWC design becomes a very complex problem. Beyond the difficulties, it is stressed the importance of developing systematic PWC methods and tools based on limited/simplified process information (e.g. steady-state models), and easy to understand for industrial acceptance. ${ }^{1}$

Many PWC methodologies have been published during the last decades. ${ }^{2}$ In Rangaiah and Kariwala ${ }^{3}$ a basic classification is proposed according to two main groups. On the one hand, PWC methods can be distinguished taking into account an approach-based criteria, where heuristics, mathematical, optimization or hybrid methods can be discriminated, see Rangaiah and Kariwala ${ }^{3}$ for a complete survey. On the other hand, a structure-based classification depending on the degree of centralization can be done, where common choices are 

multi-loop controllers are implemented. As stated in Campo and Morari, ${ }^{4}$ this type of control policy is widely used in practice, allowing an efficient and reliable process operation. This is because decentralized strategies do not require the development of complex process models. In addition, the implemented controllers can be tuned individually by selecting a few parameter values (e.g. the static gain and time constant for a proportional-integral PI controller). Other important advantages include: (i) the operating philosophy is understandable for process operators, (ii) the controllers can be easily tuned (the parameters have a direct/localized effect), and (iii) the computational load is cheap. However, conventional PID-based multiloop controllers present a significant drawback: they do not handle (actuator) constraints. Moreover, the control structure may require reconfiguration when certain actuator/sensor faults occur. Even though individual controllers can be activated/deactivated for fault handling, the number of potential fault combinations can be large and thus it is difficult to evaluate all control alternatives at the design stage. ${ }^{5}$

On the contrary, when centralized strategies are considered, then MIMO controllers (typically MPC controllers) are synthesized. As it is well known, MPC-based systems present some important advantages over multi-loop structures. MPC systems can (systematically) handle constraints on inputs (in absolute values and in differential steps) and outputs. In addition, they can manage various types of control objectives depending on the defined cost function. Therefore, it offers a powerful framework for integrating control and optimization tasks. Moreover, MPC are suitable for tolerating structural faults by monitoring real-time modifications in the populations of manipulated and controlled variables. As stated in Stephanopoulos and Reklaitis, ${ }^{6}$ given the maturation of MPC-based technologies in the last years, the classic hierarchical tasks of planning, scheduling and regulatory control can be developed such that the corresponding time scale (days/weeks to seconds/miliseconds) can be considered transparent. Beyond MPC advantages, many PWC methodologies are based on decentralized control structures and there are relatively few contributions that make use 


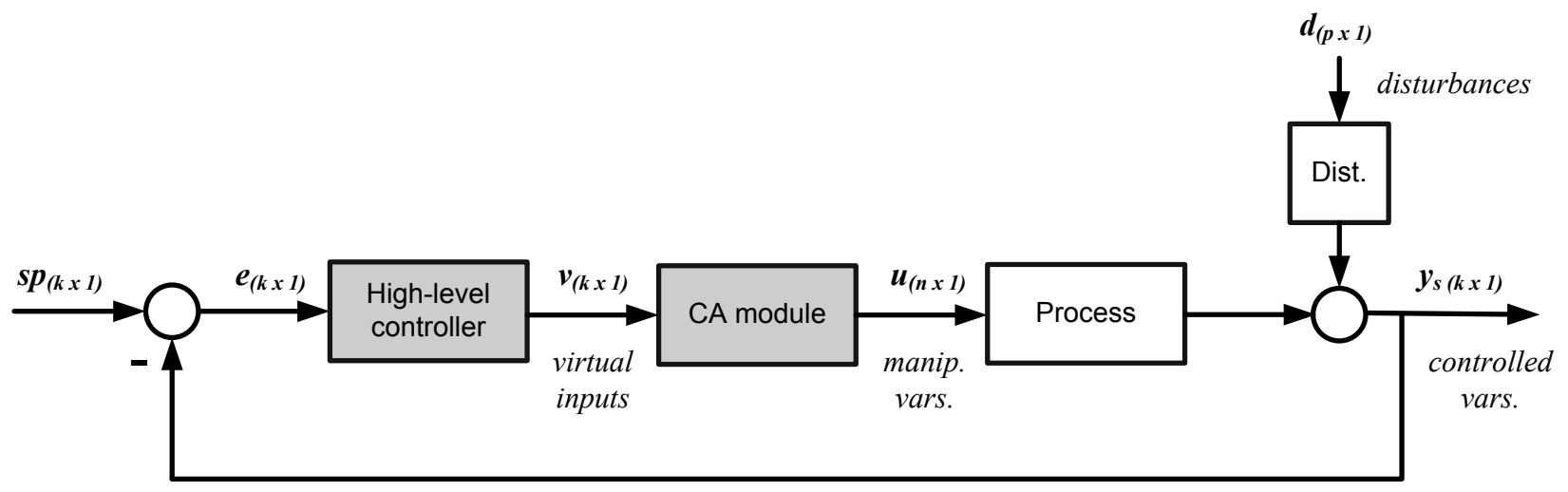

Figure 1: CA-based system architecture

of centralized or mixed strategies. The main reasons range from the process model development/identification and the implementation/maintenance costs, to the computational load characteristic of centralized control systems. ${ }^{7}$ In Rangaiah and Kariwala ${ }^{3}$ it is anticipated that near future PWC methodologies will be mainly based on mixed strategies, i.e. a combination of decentralized and centralized control.

In the last years, an alternative approach has captured the interest of the control community in different application areas, such as aerospace, ${ }^{8}$ ships, ${ }^{9}$ underwater and ground vehicles, ${ }^{10}$ among other. It considers the implementation of a basic, non-reconfigurable control scheme which defines primary control actions in order to satisfy the main process requirements. In addition, the so-called control allocation problem (CAP) must be solved jointly. ${ }^{11}$ The CAP addresses the optimal distribution of the primary control actions over the available actuators, so as to meet all pre-established objectives. A typical CA architecture is shown in Fig. 1. It includes two hierarchical levels: (i) a high-level control (HLC) strategy conceived to calculate the vector of primary control actions (also called vector of virtual inputs), and (ii) a CA module for systematically mapping the vector of virtual inputs on the manipulated variables vector. This approach presents some attractive points:

1. First, the modular architecture of Fig. 1 commonly offers some advantages: (i) the HLC design can be done by taking into account minimum information about the CA block, (ii) the CA module can naturally handle actuator constraints. It should be noted 
that the difficulty of setting constraints in the CA algorithm is not comparable with the complexity involved in including constraints on the high-level control problem. ${ }^{12}$ (iii) the CA module has the ability to accommodate faults from the automatic redistribution of the manipulated variables. In fact, it is possible to avoid a high-level control structure reconfiguration, even when a complete effectiveness loss of an actuator occurs. ${ }^{13}$ This contrasts with other control strategies where structural modifications are typically employed to respond to critical abnormal situations. ${ }^{14,15}$

2. Second, the CA block has the ability to handle failures not conceived at the design stage. It can update the control algorithm by making online decisions based on the information provided by a fault detection and diagnosis subsystem. ${ }^{11}$

3. Third, the CA approach has been successfully used in aircraft control systems in order to ensure an optimum use of the actuators. ${ }^{8}$ In addition, numerous papers related to space and marine applications have been published.

\subsection{Contribution of the work}

In this work, the CA philosophy is considered in order to develop a PWC methodology devised particularly to process control. In the framework of $\mathrm{CA}$, some proposals from the literature initially establish a partition of the available model with the objective of isolating the high-level control structure design task from the CA problem. In addition, most contributions are based on state-space models of the process. ${ }^{12,13,16}$ In this paper, the idea is to employ steady-state gain matrix models that can be obtained from simple step-tests performed on the (stabilized) process. Here, the model partition is based on an approximation of its singular value decomposition (SVD). The proposal is to discard the smallest singular values of the SVD, which means to consider only the most effective control directions of the process. ${ }^{17}$

After the model decomposition, the high-level control structure design is addressed. The 
implementation of a decentralized control structure based on conventional single-input/singleoutput PID controllers is proposed. In this context, the selection of the controlled variables, the virtual inputs and the definition of the corresponding pairings is performed through the minimization of (i) the well-known sum of squared deviations (SSD), ${ }^{18}$ and (ii) the relative gain array number (RGAn). ${ }^{17}$ This is done through a multi-objective optimization framework implemented with genetic algorithm.

In addition, the optimization algorithm for solving the CA problem must be carefully selected taking into account the chosen objective function, the considered constraints (related to the actuators model), and the computational load, among others. The works of Johansen and Fossen, ${ }^{11}$ Petersen and Bodson ${ }^{19}$ and Bodson ${ }^{20}$ result useful for evaluating different CA techniques that could be used in the context of process control. A brief state-of-theart concerning some available CA methods is included in this paper, and the adoption of the active set based solver proposed by Harkegard ${ }^{21}$ is justified by analyzing its potential performance, computational complexity and the possibilities of real-time implementation.

This paper is organized as follows: the next section presents the complete PWC design procedure, datailing the employed methodologies/tools and the corresponding implementation. Section 3 includes the evaluation of the CA-based method on the well-known Tennessee Eastman (TE) process. ${ }^{22}$ The development of the high-level structure (presented in section 3.1) together with the configuration of the CA module (detailed in section 3.2) conform the PWC structure which is utilized to control the rigorous nonlinear TE process model. A complete set of closed-loop simulations is proposed in section 3.3. In fact, two CA alternatives (namely the generalized inverse plus simple saturation and an active set method for the weighted least-squares formulation) are tested taking into account different input constraint sets. In particular, the dynamic performance and timing properties of both CA algorithms are comprehensively evaluated in order to assess its real-time implementation feasibility. The simulations consider several test scenarios which are characterized by setpoint and disturbances changes as in Downs and Vogel. ${ }^{22}$ In addition, the PWC solution presented by Molina 
Table 1: PWC design procedure.

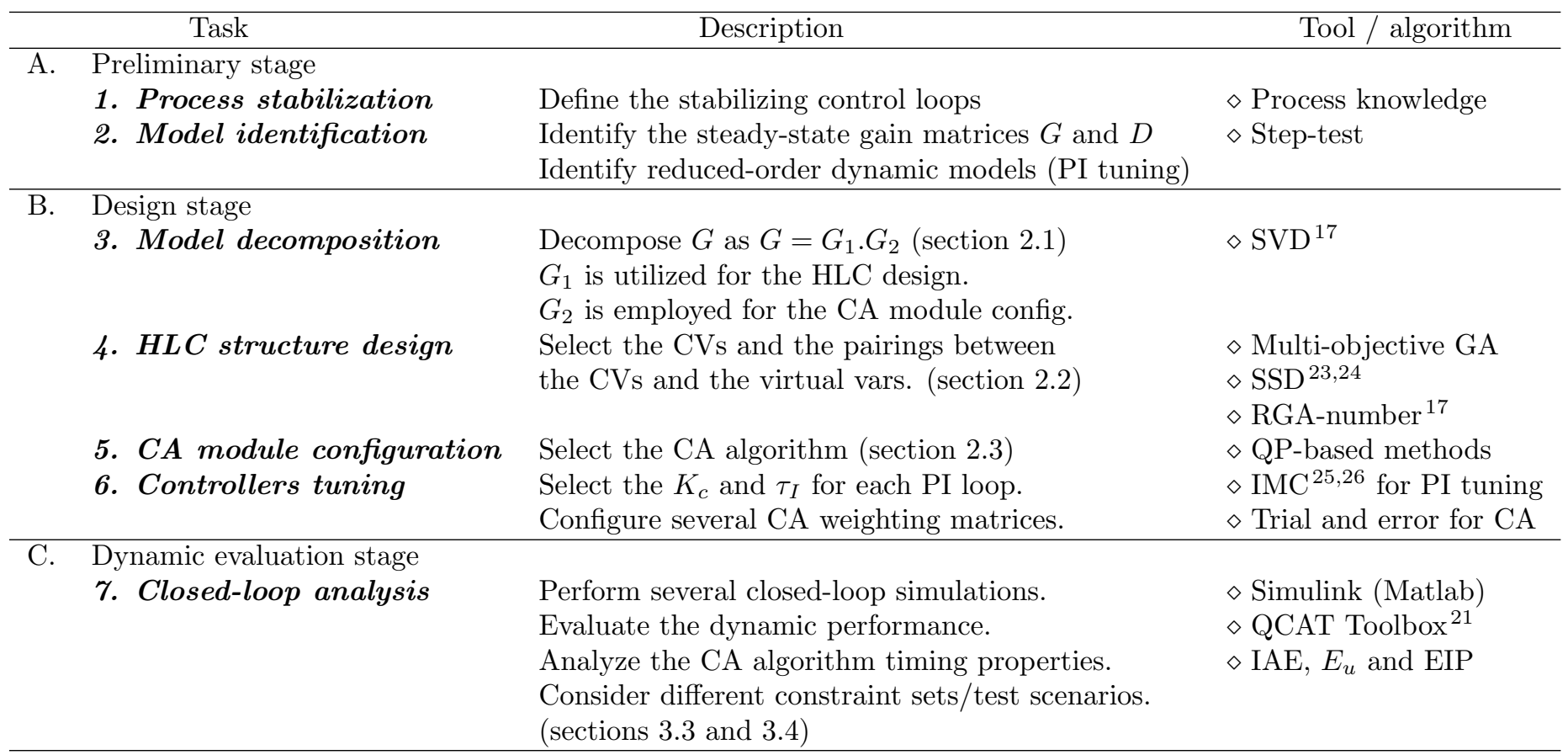

et al. ${ }^{23}$ is also simulated to compare its performance against the proposed CA solution. The final conclusions are commented in section 4.

\section{Plantwide control structure design}

The PWC design procedure is presented in this section. The complete methodology is illustrated in Table 1, where the design stage constitutes the main contribution.

Consider a (stabilized) plant with $n$ inputs, $m$ outputs and $p$ disturbances represented as:

$$
y(s)=G(s) u(s)+D(s) d(s)
$$

where $y(s)$ is the $m \times 1$ output vector, $u(s)$ the $n \times 1$ input vector, and $d(s)$ corresponds to the disturbances vector with dimension $p \times 1$. In addition, $G(s)$ and $D(s)$ are the process and disturbances transfer function matrices, respectively, with dimension $m \times n$ and $m \times p$. In the control allocation (CA) framework, the control structure involves a high-level control 
(HLC) strategy and a CA module. The corresponding architecture is illustrated in Fig. 1.

The objective of the HLC strategy is to calculate a vector of virtual inputs $v(s)$ of dimension $k \times 1$. Normally, the $k$ virtual variables are defined taking into account the main process requirements to be satisfied, ${ }^{11}$ e.g. production rate, product quality, etc. in the context of process control. Specifically, if $q$ represents the number of process outputs that must be indefectibly controlled, then $k$ must be chosen such that $k \geq q$.

The CA module is responsible of managing the manipulated variables $u(s)$ such that their combined effect be equivalent to that of the virtual inputs $v(s) .{ }^{11}$ The $\mathrm{CA}$ module is based on a model of the form:

$$
v=f(u), \quad u \in \mathcal{U}
$$

where $f(u)$ is a vector of linear/nonlinear functions of $u$, and $\mathcal{U}$ represents the control constraints. Due to the constraints, a feasible $u \in \mathcal{U}$ could not exist and the CA algorithm should compute a solution that minimizes the allocation error $v-f(u)$. However, given that the number of available actuators is commonly greater than the number of outputs that must be controlled (i.e. $n>k$ ), the solution to the problem of obtaining $u \in \mathcal{U}$ from $v$ may not be unique. This context results auspicious to formulate the CA problem in an optimization framework, in which secondary control objectives can be incorporated. ${ }^{11}$ This issue is discussed in section 2.3.

In this work, the following linear CA model is assumed:

$$
v=M u, \quad u \in \mathcal{U}
$$

where $M$ is a $k \times n$ matrix usually called the control effectiveness matrix. Up to this point, some important questions appear related to the control structure design: How are the virtual variables $v$ defined? What (steady-state) information should be used to design the HLC structure? How is the control effectiveness matrix $M$ defined? These answers and the complete design procedure is detailed step by step in the following sections. 


\subsection{Decomposition of the G matrix}

As it is known, the singular value decomposition (SVD) allows an exact representation of any matrix. For a (complex) $m \times n$ matrix $G$, the SVD is defined as: ${ }^{17}$

$$
G=U \Sigma V^{T}
$$

where $U$ is a $m \times m$ unitary matrix of output singular vectors, $V$ is a $n \times n$ unitary matrix of input singular vectors, and the $m \times n$ matrix $\Sigma$ contains $l=\min (m, n)$ (real) non-negative singular values $\sigma_{i}$ arranged in a descending order along its diagonal. Assume that the SVD of $G$ is partitioned as:

$$
G=\left[\begin{array}{ll}
U_{1} & U_{2}
\end{array}\right]\left[\begin{array}{cc}
\Sigma_{1} & 0 \\
0 & \Sigma_{2}
\end{array}\right]\left[\begin{array}{c}
V_{1}^{T} \\
V_{2}^{T}
\end{array}\right]
$$

where $\Sigma_{1}$ contains the $k$ largest singular values, and $\Sigma_{2}$ includes the $l-k$ smallest singular values. In addition, $U$ and $V$ are partitioned accordingly with $\Sigma_{1}$ and $\Sigma_{2}$. In particular, forcing to zero the $l-k$ singular values of $\Sigma_{2}$ produces an approximate representation of $G$. This corresponds to a dimensionality reduction of the SVD matrices because $U_{2}$ and $V_{2}$ can be discarded: ${ }^{27}$

$$
G \cong U_{1} \Sigma_{1} V_{1}^{T}
$$

Here, $U_{1}, \Sigma_{1}$ and $V_{1}$ have dimension $m \times k, k \times k$ and $n \times k$, respectively. From a control perspective, to discard the smallest singular values of $\Sigma$ means not considering the least effective control directions of the process. ${ }^{17}$

Taking into account Eq. 6, the approximate representation of $G$ can be expressed as:

$$
G \cong G_{1} G_{2}
$$

with:

$$
G_{1}=U_{1}
$$




$$
G_{2}=\Sigma_{1} V_{1}^{T}
$$

where $G_{2}$ has dimension $k \times n$.

From now, suppose that the CA problem of Eq. 3 is based on $G_{2}$ :

$$
v=G_{2} u, \quad u \in \mathcal{U}
$$

Assuming that $G_{2}$ has full rank and neglecting any constraint on $u$, it can be shown that:

$$
u=G_{2}^{+} v
$$

where:

$$
G_{2}^{+}=G_{2}^{T}\left(G_{2} G_{2}^{T}\right)^{-1}
$$

Working with Eqs. 9 and 12 yields:

$$
G_{2}^{+}=V_{1} \Sigma_{1}^{-1}
$$

Therefore,

$$
u=V_{1} \Sigma_{1}^{-1} v
$$

which corresponds to the explicit solution of the (unconstrained) linear CA problem $v=G_{2} u$. Taking into account Eqs. 6 and 14 it is easy to show that the transfer function that relates $y$ with $v$ results:

$$
y=U_{1} v
$$

Conversely, suppose that $y$ and $v$ are related through $G_{1}=U_{1}$, then:

$$
y(s)=U_{1}(s) v(s)+D(s) d(s)
$$




\subsection{High-level control structure design}

In this work it is proposed the development of a decentralized (diagonal) high-level control structure, implemented with single-input single-output PID controllers. Thus, three main tasks must be analyzed:

1. the selection of $k$ controlled variables (CVs) from $m$ outputs,

2. the pairing selection between the $k \mathrm{CVs}$ and the $k$ virtual variables $v$,

3. the tuning of the $k$ PID controllers.

In the following, two well-known scalar indexes are proposed for supporting tasks 1 and 2 . 
Consider the steady-state (stabilized) process models represented by the matrices $G$ (input-output) and $D$ (disturbances-outputs). Taking into account the matrix decomposition presented in Eq. 7, then it can be expressed:

$$
y=G_{1} v+D d
$$

where all vectors/matrices were defined previously. Eq. 19 can be partitioned as:

$$
\left[\begin{array}{l}
y_{s} \\
y_{r}
\end{array}\right]=\left[\begin{array}{l}
G_{1 s} \\
G_{1 r}
\end{array}\right] v+\left[\begin{array}{c}
D_{s} \\
D_{r}
\end{array}\right] d
$$

Here, $G_{1 s}$ corresponds to the square $k \times k$ subprocess to be controlled, based on the selection of $k$ outputs from $G_{1}$. Accordingly, $G_{1 r}, D_{s}$ and $D_{r}$ have dimension $(m-k) \times k, k \times p$ and $(m-k) \times p$, respectively. From the Internal Model Control (IMC) theory and assuming steady-state perfect control (i.e. $\left.y_{s}=y_{s}^{s p}\right)$, then: ${ }^{18,24}$

$$
y_{r}=\left[G_{1 r} G_{1 s}^{-1}\right] y_{s}^{s p}+\left[D_{r}-G_{1 r} G_{1 s}^{-1} D_{s}\right] d
$$

The above expression denotes the steady-state deviations of the uncontrolled variables $y_{r}$ from their operating points, when setpoint and disturbances changes are considered. In this context, the sum of square deviations (SSD) is defined as: ${ }^{23,24}$

$$
S S D=\left\|G_{1 r} G_{1 s}^{-1}\right\|_{F}^{2}+\left\|D_{r}-G_{1 r} G_{1 s}^{-1} D_{s}\right\|_{F}^{2}
$$

where $\|\ldots\|_{F}$ represents the Frobenius norm.

Then, consider a particular selection of $k$ outputs represented by $G_{1 s}$. Additionally, consider a diagonal pairing for $G_{1 s}$. In this context, the $R G A$ number (RGAn) is defined as: ${ }^{17}$

$$
R G A n=\left\|\Lambda\left(G_{1 s}\right)-I_{k}\right\|_{s u m}
$$


where $\|\ldots\|_{\text {sum }}$ represents the sum norm, $I_{k}$ the $k \times k$ identity matrix and $\Lambda$ the (steadystate) relative gain array (RGA).

Therefore, the optimal selection of $k$ outputs together with the corresponding $k$ pairings based on the minimization of the $S S D$ and $R G A n$ indexes can be formulated as:

$$
\min _{(J)}\left[S S D_{(J)}, R G A n_{(J)}\right]
$$

subject to:

$$
\begin{gathered}
\operatorname{det}\left[G_{1 s_{(J)}}\right] \neq 0 \\
\Lambda_{i i}\left[G_{1 s_{(J)}}\right]>0, \quad i=1, \ldots, k \\
\operatorname{Re}\left\{\lambda_{i}\left[G_{1 s_{(J)}}\left(G_{1 s_{(J)}} \bigotimes I\right)^{-1}\right]\right\}>0, \quad i=1, \ldots, k
\end{gathered}
$$

Here, the constraints of Eqs. 25 and 26 are used to discard non-feasible solutions, where $\Lambda_{i i}$ represents the diagonal elements of the (rearranged) RGA. In addition, the inequality of Eq. 27 corresponds to the stability criterion proposed by Garcia and Morari, ${ }^{26}$ where $\lambda_{i}$ is the i-th eigenvalue and $\otimes$ represents the element-by-element product. Finally, $J=$ $\left\{j_{1}, j_{2}, \ldots, j_{k}\right\} \subset\{1,2, \ldots, m\}$ corresponds to an index set which parameterizes the selection of the controlled variables.

On the one hand, the minimization of the SSD leads to maximize the minimum singular value of the selected $G_{1 s}$. Within the possibilities, this implies a well-conditioned $G_{1 s}$ which favors the system controllability. ${ }^{23}$ On the other hand, when the RGAn is minimized the rearranged $G_{1 s}$ presents a RGA which is as close as possible to the identity matrix.

\subsubsection{Implementation}

In this paper, the multi-objective optimization problem of Eq. 24 is solved through the Matlab function gamultiobj, which is based on genetic algorithm (GA). This optimization procedure is adequate for large combinatorial problems, and besides presents low likehood 
of obtaining local optima. After each generation, the GA tends to minimize the functional costs values, trying to keep a high diversity of solutions. Here, a vectorized version of the gamultiobj is implemented in order to accelerate computation times.

For each proposed chromosome (i.e. candidate solution) the pairing problem is solved through the algorithm developed by Kariwala and Cao. ${ }^{29}$ Their procedure is based on a branch and bound method which minimizes two selection criteria: the RGA number and the $\mu$ interaction measure. For each obtained Pareto set, the multi-objective GA retains the pairing which minimizes the RGA number.

Finally, it is important to clarify that the proposed GA is able to perform the selection of $k$ virtual variables (along with the $k \mathrm{CVs}$ ) when the number of considered singular values is greater than $k$. This is useful for cases where two or more singular values present similar values, thus the selection of the virtual variables is based on the minimization of the SSD and RGAn. From the controller implementation point of view, the (intermediate) unselected virtual variables must be considered as constant inputs for the control allocation module.

\subsection{Control allocation module configuration}

Several CA approaches have been proposed in the literature, see Johansen and Fossen ${ }^{11}$ for an excellent survey. In the simple saturation approach, $u$ is firstly computed through an unconstrained CA method (e.g. generalized inverses, see Eqs. 11 to 14) and then saturated to satisfy the constraints. Clearly, this procedure does not guarantee that the allocation error is annulled whenever possible, or minimized in some sense. Different constrained CA methods like the redistributed pseudo-inverse (RPI), the daisy chaining (DC) and the direct allocation method were developed in order to obtain better solutions. Although they are simple and effective, notorious sub-optimal solutions can result from the RPI and DC implementations. Moreover, the direct allocation method becomes complex when the dimension of $u$ is large. ${ }^{11}$

In this context, several approaches which explicitly minimize the (weighted) control allocation error can be encountered in the literature. They are mostly based on constrained 

2

linear programming (LP) or quadratic programming (QP), and solved using iterative algorithms. While these methods are able to converge within a finite number of iterations, generally a sub-optimal solution is implemented given the limited time available in real-time applications. Typically, the simplex method is utilized as numerical method for LP due to its reduced computational complexity. ${ }^{20}$ As commented in Johansen and Fossen, ${ }^{11}$ optimal LP solutions are located at the feasible set vertices and this translates into the use of a reduced number of actuators $u$. On the contrary, QP-based methods tends to harmonize the use of actuators and therefore are preferred for its use in CA algorithms. ${ }^{19}$

In the context of $\mathrm{CA}$, different QP-based methods such as active set or interior point are usually employed. ${ }^{19,21}$ In particular, interior point methods perform well for large-scale problems. However, they commonly require several iterations to converge because the initialization is done with points located near the center of the feasible region. On the contrary, active set methods are very efficient for CA when a good estimate of the optimal active set is available. In CA, a good starting point is generally given by the active set corresponding to the solution of the previous sampling period. In practice, the optimization problem does not change much between consecutive sampling periods and thus the required number of iterations results generally small. Active set methods are iterative methods that improve the cost function value after each iteration. Moreover, they are relatively easy to implement with low computational burden.

In this work, the CA problem is posed according to the following weighted least-squares (WLS) formulation: ${ }^{21}$

$$
\min _{u}\left\|W_{u}\left(u-u_{p}\right)\right\|+\gamma\left\|W_{v}\left(G_{2} u-v\right)\right\|
$$

subject to:

$$
\underline{u} \leq u \leq \bar{u}
$$

Here, $u_{p}$ represents the preferred control input, and $W_{u}$ and $W_{v}$ are positive definite weighting matrices. $W_{u}$ affects the control distribution among the actuators, and $W_{v}$ affects the prior- 
itization among the virtual control components. In addition, $\gamma$ corresponds to a weighting factor that relatively prioritizes the minimization of the control allocation error (primary objective) and the movement of the actuators (secondary objective). Finally, $\underline{u}$ and $\bar{u}$ represent lower and upper bounds related to position and rate limits of the actuators.

In order to obtain the optimal solution of the above CA problem, the active set based solver proposed by Harkegard ${ }^{21}$ is utilized in this study, which in turn is available in C compiled language. This solver is part of a complete CA Matlab toolbox implemented by Harkegard. ${ }^{21}$ It consists of several QP allocation functions such as wls-alloc, which solves the CA problem for a virtual control input $v$ given $G_{2}, \underline{u}, \bar{u}, W_{u}, W_{v}$ and $u_{p}$. In addition, the function $q p$-sim simulates the time response of the CA module to arbitrary inputs, measuring the computation time per sample. Moreover, a Simulink library is included so as to implement the module for QP control allocation.

In the following section, the dynamic performance and the timing properties of the active set based algorithm which operates in conjunction with the high-level controller is evaluated by considering a recognized case study. 


\section{Case study: Tennessee Eastman process}

In this section, the Tennessee Eastman (TE) case study is proposed in order to apply the design procedure based on the control allocation philosophy. For this chemical process, typical objectives are posed which must be accomplished by the plantwide control: ${ }^{22}$ (i) to recover quickly from setpoint changes and disturbances, (ii) to keep operating conditions within specific constraints, see Tables 3 and 6 from Downs and Vogel, ${ }^{22}$ (iii) to minimize the movement of valves. In this study it is assumed that the process operates at mode 1, with a $\mathrm{G} / \mathrm{H}$ mass ratio of 50/50 and a production rate of $7038 \mathrm{kgh}^{-1} \mathrm{G}$ and $7038 \mathrm{kgh}^{-1} \mathrm{H}$. In addition, two main disturbances such as $i d v(1)$ and $i d v(2)$ (see Table 2) are considered.

The TE process is open-loop unstable, thus a minimum number of control loops is necessary to stabilize the plant. Due to their integrative behavior, these control loops are typically related to level in tanks and vessels. McAvoy ${ }^{30}$ and Arkun and Downs ${ }^{31}$ proposed several methods in order to achieve the process stabilization. In this context, the levels in the reactor $x m e(8)$, the product separator $x m e(12)$, the stripper $x m e(15)$ and the reactor cooling water outlet temperature $x m e(21)$ must be controlled so as to stabilize the TE process. The corresponding control loops were implemented as in Molina et al. ${ }^{23}$

The normalized steady-state gain matrices $G$ and $D$ estimated in Molina et al. ${ }^{23}$ (corresponding to inputs-outputs and disturbances-outputs, respectively) are considered as the

Table 2: Plantwide control design: available variables.

\begin{tabular}{llll}
\hline Output & \multicolumn{1}{c}{ Description } & Input & \multicolumn{1}{c}{ Description } \\
\hline$x m e(5)$ & Recycle flow (stream 8) & $x m v(1)$ & D feed flow (stream 2) \\
$x m e(6)$ & Reactor feed rate (stream 6) & $x m v(3)$ & A feed flow (stream 1) \\
$x m e(9)$ & Reactor temp. & $x m v(4)$ & A and C feed flow (stream 4) \\
$x m e(11)$ & Product separator temp. & $x m v(5)$ & Compressor recycle valve \\
$x m e(13)$ & Product separator pressure & $x m v(6)$ & Purge valve (stream 9) \\
$x m e(16)$ & Stripper pressure & $x m v(9)$ & Stripper steam valve \\
$x m e(18)$ & Stripper temp. & $x m e(21) s p$ & Reactor cooling water outlet temp. setpoint \\
$x m e(20)$ & Compressor work & $x m v(11)$ & Condenser cooling water flow \\
$x m e(7)$ & Reactor pressure & & \\
$x m e(17)$ & Stripper underflow (stream 11) & $i d v(1)$ & A/C feed ratio (stream 4) \\
$x m e(30)$ & B comp. purge (stream 9) & $i d v(2)$ & B composition (stream 4) \\
$x m e_{G / H}$ & G/H comp. ratio (stream 11) & & \\
\hline
\end{tabular}



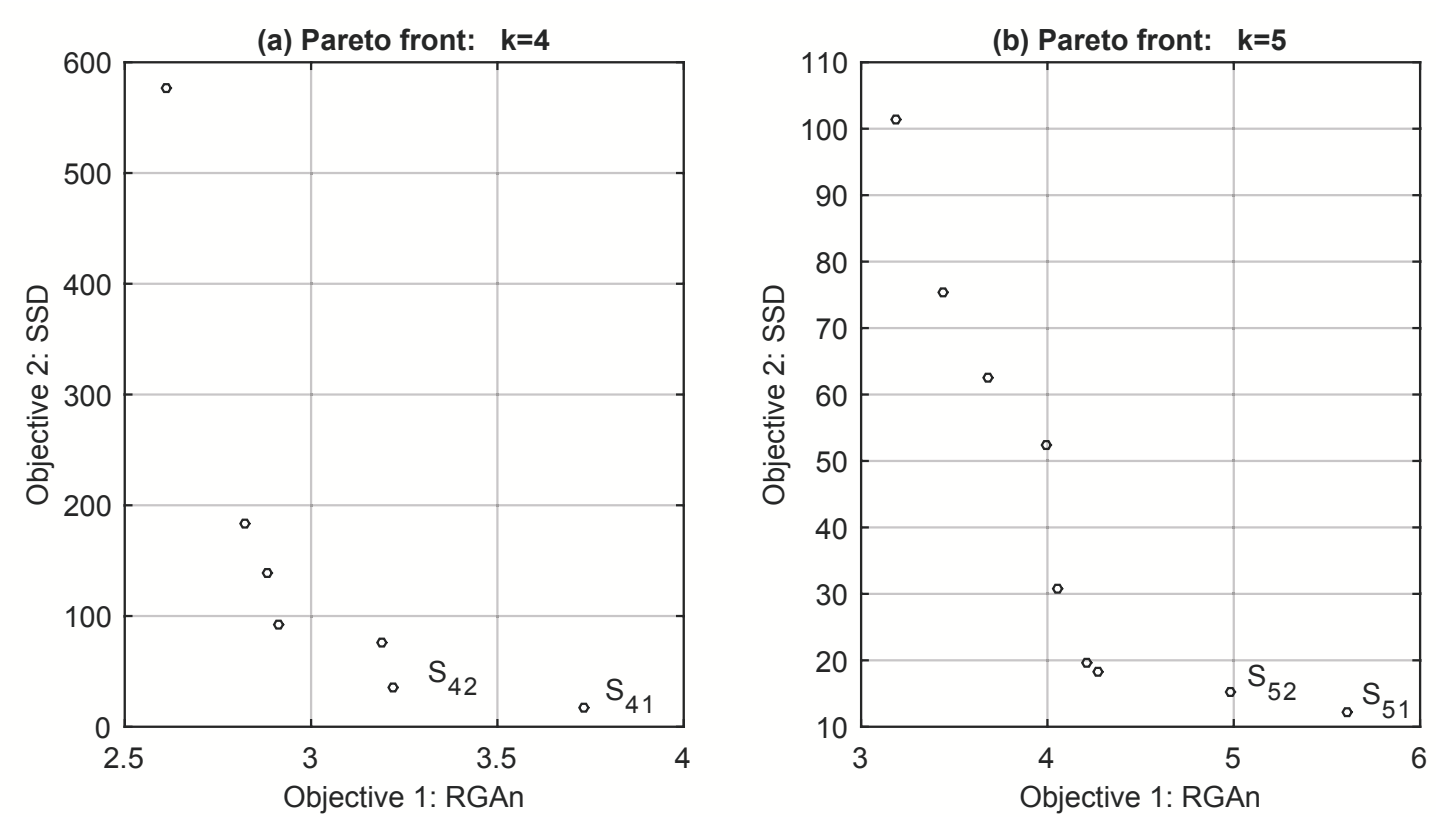

Figure 2: Alternative high-level control structures: (a) $k=4$; (b) $k=5$

starting point for the design procedure presented in section 2. The associated inputs and outputs are specified in Table 2. Note that the linear models are only valid surrounding the considered process operating point. However, if it is desired to operate the TE process at a different operating point, then the PWC methodology must be carried out taking into account the construction of new linearized models. This probably entails (significant) modifications in the nominal control structure design.

In the following sections, the design of the high-level control structure as well as the CA block configuration are presented.

\subsection{High-level control structure}

This section details the design procedure corresponding to the high-level controller, which consists of a decentralized (diagonal) structure.

In order to satisfy the TE process requirements, and also considering the setpoint tests suggested by Downs and Vogel, ${ }^{22}$ then the outputs $x m e(7), x m e(17), x m e(30)$ and $x m e_{G / H}$ must be always controlled. In this context, $k \geq 4$ must be configured. Hence, two alternative 
designs were proposed, with $k=4$ and $k=5$. The multi-objective genetic algorithm (MOGA) was executed twice so as to solve the problem stated in Eqs. 24-27 for each case. The main MOGA parameters are detailed in Table S1 (see Supporting Information, Appendix A). The obtained solution sets corresponding to $k=4$ and $k=5$ are shown in Figs. 2(a) and 2(b), respectively. The Pareto optimal set consists of 7 solutions for $k=4$, and 9 solutions for $k=5$.

Note that the high-level control structure design could be based on the exclusive minimization of the SSD index, see for example Luppi et al. ${ }^{15}$ But, in this work the minimization of a second objective (i.e. the RGA-number) was considered because $G_{1 s}$ (i.e. the selected subprocess to be controlled) typically presents considerable interactions. In fact, the diagonal RGA elements corresponding to $S_{41}$ are $0.59,0.47,0.39$ and 0.71 . In the context of multi-objective optimization, one option is to select the final solution based on heuristic criteria. ${ }^{32}$ However, alternative methods exist in order to select an optimal Pareto solution. ${ }^{33}$ In this study, the solution $S_{41}$ detailed in Table 3 was selected as the final high-level structure because: (i) considering $k=4$ solutions, the $S_{41}$ has the lowest SSD and acceptable RGA matrix (the remaining Pareto solutions do not significantly improve the RGA quality, but increase the SSD), (ii) contrasting with $k=5$ solutions, $S_{41}$ presents a comparable SSD value and RGA quality. Moreover, $S_{41}$ contemplates the use of four control loops instead of five. A major issue is that $S_{41}$ behaves satisfactorily when it is implemented and simulated subject to several test scenarios, see sections 3.3 and 3.4 .

Some $k=4$ and $k=5$ solutions are presented in Table 3. For each solution, it shows the chosen virtual and controlled variables, the corresponding loop pairings, and their SSD and RGA-number values. As commented in section 2.2.1, the selection of a particular $k$ does not necessarily imply the selection of the first $k$ columns of $G_{1}$. However, note that the obtained Pareto optimal solutions mainly involve those virtual variables with the most control power, i.e. $v(1), v(2), v(3)$, etc. The number that identifies the virtual variable refers to the column number of the matrix $G_{1}$. Fig. 3 and Fig. S1 (see Supporting Information, Appendix D) 
Table 3: Alternative high-level control structures

\begin{tabular}{ccccc}
\hline & $S_{41}$ & $S_{42}$ & $S_{51}$ & $S_{52}$ \\
\hline & $v(1)-x m e(7)$ & $v(1)-x m e(7)$ & $v(1)-x m e(7)$ & $v(1)-x m e(11)$ \\
& $v(2)-x m e(17)$ & $v(3)-x m e_{G / H}$ & $v(2)-x m e(17)$ & $v(2)-x m e(7)$ \\
& $v(3)-x m e_{G / H}$ & $v(4)-x m e(30)$ & $v(3)-x m e_{G / H}$ & $v(3)-x m e_{G / H}$ \\
& $v(4)-x m e(30)$ & $v(6)-x m e(17)$ & $v(4)-x m e(30)$ & $v(4)-x m e(30)$ \\
& & & $v(6)-x m e(18)$ & $v(6)-x m e(17)$ \\
\hline$k:$ & 4 & 4 & 5 & 5 \\
SSD: & 17.70 & 35.42 & 12.31 & 15.33 \\
RGA-number: & 3.73 & 3.22 & 5.61 & 4.98 \\
\hline
\end{tabular}

show implementation details of $S_{41}$ and $S_{42}$.

Finally, Table S2 (see Supporting Information, Appendix B) summarizes the tuning parameters of the four PI control loops corresponding to solution $S_{41}$. As in Luppi et al., ${ }^{34}$ these parameters were obtained through the Internal Model Control (IMC) method proposed by Rivera. ${ }^{25}$ It is based on the application of some system identification procedure over the process. Here, a step-test were performed in the Matlab environment taking into account the rigorous nonlineal TE model. For this experiment, a simplified version of the CA block based on unconstrained lineal CA was employed. The main information needed to configure this block was the $G_{2}$ matrix. This is valid given the relatively small magnitude of the steps applied to the virtual variables $v$. In fact, the applied steps did not move considerably the manipulated variables $u$ with respect to the operating point. For this reason, the constraints were not activated in any case.

In sections 3.3 and 3.4, the dynamic performance of the final control structure detailed in Fig. 3 (i.e. $S_{41}$ together with different configurations of the CA block) is analyzed. 


\subsection{Control allocation block}

Continuing with the control structure design, two alternative algorithms for the CA block are proposed. They are based on the approaches commented in section 2.3: (i) generalized inverse (GI) plus simple saturation, and (ii) active set method for the weighted least-squares formulation (WLS). The objective is to compare the dynamic performance of the produced solutions as well as their timing properties in order to assess the real-time implementation feasibility. Both CA alternatives are analyzed taking into account two cases:

1. The original constraint set detailed in Table 3 in Downs and Vogel, ${ }^{22}$ which is considered here as a non-severe constraint set. As will be shown in section 3.4.1, for this case the constraints are not activated in any simulation scenario (see simulations $\operatorname{Sim}_{1}$ to $\operatorname{Sim}_{7}$ ). In this context, the study will focus on the advantages of the CA to handle secondary objectives, in particular the reduction of the control energy. With this purpose, the tuning of the gamma $(\gamma)$ parameter for the WLS is presented in section 3.4.1.

2. Some adjusted versions of the original constraint set which are defined in section 3.4 .2 (named here as severe constraint sets). They consist on specific position and/or rate limits such that they are activated in certain simulation scenarios (see simulations $\mathrm{Sim}_{8}$ to $\mathrm{Sim}_{13}$, section 3.4.2). The aim is to analyze the potential benefits provided by the WLS algorithm, which explicitly considers the constraints. 
Table 4: Proposed simulations

\begin{tabular}{|c|c|c|c|}
\hline Simulation & Scenario & CA algorithm & Constraint Set \\
\hline Sim $_{1}$ & $S c_{7}$ & WLS, $\gamma=1 \times 10^{6}$ & Nominal \\
\hline $\mathrm{Sim}_{2}$ & $S c_{7}$ & WLS, $\gamma=10$ & $"$ \\
\hline $\mathrm{Sim}_{3}$ & $S c_{7}$ & WLS, $\gamma=1$ & $"$ \\
\hline $\mathrm{Sim}_{4}$ & $S c_{1}$ & WLS, $\gamma=10$ & $"$ \\
\hline $\operatorname{Sim}_{5}$ & $S c_{2}$ & WLS, $\gamma=10$ & $"$ \\
\hline $\operatorname{Sim}_{6}$ & $S c_{4}$ & WLS, $\gamma=10$ & $"$ \\
\hline $\mathrm{Sim}_{7}$ & $S c_{3}$ & WLS, $\gamma=10$ & " \\
\hline $\operatorname{Sim}_{8}$ & $S c_{6}$ & GI & Set 1 \\
\hline $\operatorname{Sim}_{9}$ & $S c_{6}$ & WLS, $\gamma=1 \times 10^{6}$ & Set 1 \\
\hline $\operatorname{Sim}_{10}$ & $S c_{5}$ & GI & Set 2 \\
\hline $\operatorname{Sim}_{11}$ & $S c_{5}$ & WLS, $\gamma=1 \times 10^{6}$ & Set 2 \\
\hline $\operatorname{Sim}_{12}$ & $S c_{7}$ & GI & Set 3 \\
\hline $\operatorname{Sim}_{13}$ & $S c_{7}$ & WLS, $\gamma=1 \times 10^{6}$ & Set 3 \\
\hline
\end{tabular}

Next section presents a complete set of simulations concerning the TE case study and the proposed plantwide control structure.

\subsection{Simulations}

This part of the paper describes the realization of various simulations of the complete system, including the rigorous nonlinear TE process model controlled with the high-level structure plus the CA block, see Fig. 3. The aim is to evaluate the dynamic performance taking into account alternative CA configurations and constraint sets, subject to several test scenarios characterized by setpoint changes and disturbances. In addition, a previous solution presented by Molina et al. ${ }^{23}$ was simulated for the sake of comparison.

Table 4 details the set of performed simulations. They can be grouped according to the considered constraint set: (i) nominal constraints (simulations $\operatorname{Sim}_{1}$ to $\operatorname{Sim}_{7}$ ), (ii) severe constraints (simulations Sim $_{8}$ to Sim $_{13}$ ). These cases are analyzed in sections 3.4 .1 and 3.4.2, respectively. Table 5 specifies the setpoints and disturbances scenarios. In addition, the severe constraint sets are specified in Table 6 . The parameters corresponding to the CA algorithms are detailed in Tables S3-S4 (Supporting Information, Appendix C).

In the following, several scalar indexes are presented to quantify the dynamic performance of the solutions: 
Table 5: Simulation scenarios: setpoints and disturbances

\begin{tabular}{cclccc}
\hline Scenario & Time [h.] & \multicolumn{1}{c}{ Event } & Process var. & Type & Magnitude \\
\hline$S c_{1}$ & 10 & Reactor op. pressure change & $x m e(7)$ & step & $-3 \%$ \\
$S c_{2}$ & 10 & Production rate change & $x m e(17)$ & step & $-5 \%$ \\
$S c_{3}$ & 10 & Purge gas B comp. change & $x m e(30)$ & step & $+14 \%$ \\
$S c_{4}$ & 10 & Product mix change & $x m e_{G / H}$ & step & $50 \mathrm{G} / 50 \mathrm{H}$ to $45 \mathrm{G} / 55 \mathrm{H}$ \\
$S c_{5}$ & $12-14$ & A/C feed ratio, B comp. const. (str. 4) & $i d v(1)$ & step & enabled \\
$S c_{6}$ & $11-25$ & B comp., A/C ratio constant (str. 4) & $i d v(2)$ & step & enabled \\
$S c_{7}$ & $12-14$ & A/C feed ratio, B comp. const. (str. 4) & $i d v(1)$ & step & enabled \\
& $11-25$ & B comp., A/C ratio constant (str. 4) & $i d v(2)$ & step & enabled \\
\hline
\end{tabular}

Table 6: Severe constraint sets

\begin{tabular}{llllll}
\hline $\begin{array}{l}\text { Constraint } \\
\text { set }\end{array}$ & Input & $\begin{array}{l}\text { Position } \\
\text { low limit }[\%]\end{array}$ & $\begin{array}{l}\text { Position } \\
\text { high limit }[\%]\end{array}$ & $\begin{array}{l}\text { Rate } \\
\text { low limit }[\% / \mathrm{h}]\end{array}$ & $\begin{array}{l}\text { Rate } \\
\text { high limit }[\% / \mathrm{h}]\end{array}$ \\
\hline Set 1 & $x m v(6)$ & 0 & 50 & - & - \\
Set 2 & $x m v(1)$ & 0 & 100 & -0.45 & +0.45 \\
Set 3 & $x m v(1)$ & 61 & 100 & -0.45 & +0.45 \\
& $x m v(6)$ & 0 & 50 & -0.53 & +0.53 \\
\hline
\end{tabular}

1. Integral absolute error (IAE):

$$
I A E=\int_{t_{1}}^{t_{2}}|r(t)-y(t)| d t
$$

2. Error improvement percent (EIP):

$$
E I P=\frac{I A E^{\text {base }}-I A E^{\text {new }}}{I A E^{\text {base }}} 100
$$

3. Control energy $\left(E_{u}\right)$ :

$$
E_{u}=\int_{t_{1}}^{t_{2}}\left[u(t)-u_{0}\right]^{2} d t
$$

where $r(t)$ represents the setpoint, $y(t)$ the system output, base refers to some solution proposed as reference, and new represents the solution to be evaluated. In addition, $u(t)$ is the control signal, $u_{0}$ the corresponding nominal value (operating point), and $\left[t_{1}, t_{2}\right]$ the evaluation time period. Note that the EIP index can also be computed taking into account the control energy.

As the dynamic disturbance sensitivity (DDS) metric proposed by Konda and Ranga- 
iah, ${ }^{35}$ the IAE and $E_{u}$ represent comprehensive measures which include the information on process variables during transient responses generated by disturbances and setpoint changes. Moreover, they share several advantages such as: (i) the computation procedure remains the same irrespective of the control structure, (ii) they can be computed easily using rigorous process simulators, and (iii) they facilitate the early detection of instability, among other. In this work, the IAE index was computed for all the process outputs recommended by Downs and Vogel ${ }^{22}$ (section Dynamic performance comparisons). Finally, the $E_{u}$ index was computed for all the manipulated variables, except for those involved in the stabilizing control loops, namely $x m v(2), x m v(7), x m v(8)$ and $x m v(10)$, see Molina et al. ${ }^{23}$ 


\subsection{Results}

\subsubsection{Case 1: nominal constraints}

The first analysis corresponds to a dynamic performance comparison taking into account different gamma $(\gamma)$ values for the CA block based on the WLS algorithm. The utilized test scenario corresponds to $S c_{7}(i d v(1)$ and $i d v(2)$, see Table 5). Table 7 shows the IAE, EIP and $E_{u}$ values related to simulations $\operatorname{Sim}_{1}, \operatorname{Sim}_{2}$ and $\mathrm{Sim}_{3}$. These indexes were computed for key process variables as suggested by Downs and Vogel, ${ }^{22}$ where Op. costs refers to the operating costs for the TE process. ${ }^{22}$

In general, the configuration of relatively small values of $\gamma\left(\right.$ much smaller than $1 \times 10^{6}$ ) aims to provide greater importance to the secondary control objective, i.e. to minimize the movement of the manipulated variables. As a consequence of this, the error $B u-v$ increases affecting the dynamic performance of the system. In this context, the $\gamma$ value should be adjusted to obtain a suitable trade-off between dynamic quality and control energy

Table 7: Dynamic performance comparison. Alternative $\gamma$ values for the WLS approach. Simulation scenario: $S c_{7}(i d v(1)$ and $i d v(2))$

\begin{tabular}{|c|c|c|c|}
\hline $\begin{array}{c}\text { Index: } \\
\text { CA config.: } \\
\text { Simulation(s): }\end{array}$ & $\begin{array}{c}\text { IAE } \\
\text { WLS }\left(\gamma=1 \times 10^{6}\right) \\
\operatorname{Sim}_{1}\end{array}$ & $\begin{array}{c}\text { EIP [\%] } \\
\text { WLS }(\gamma=10) \\
\text { Sim }_{1} \text { vs. Sim } 2\end{array}$ & $\begin{array}{c}\text { EIP }[\%] \\
\text { WLS }(\gamma=1) \\
\text { Sim }_{1} \text { vs. } \text { Sim }_{3}\end{array}$ \\
\hline$x m e(1)$ & $2.4 \times 10^{3}$ & 34.8 & 84.9 \\
\hline$x m e(2)$ & $8.9 \times 10^{6}$ & 39.6 & -25.9 \\
\hline$x m e(3)$ & $2.4 \times 10^{7}$ & 1.1 & -12.2 \\
\hline$x m e(4)$ & $1.2 \times 10^{4}$ & 11.8 & -12.2 \\
\hline$x m e(7)$ & $1.0 \times 10^{7}$ & -20.9 & -25.3 \\
\hline$x m e(17)$ & $2.9 \times 10^{4}$ & 16.2 & 25.8 \\
\hline$x m e(30)$ & $3.7 \times 10^{5}$ & -14.5 & -76.8 \\
\hline$x m e_{G / H}$ & $5.0 \times 10^{3}$ & 16.0 & -57.9 \\
\hline Op. costs & $3.6 \times 10^{7}$ & 3.6 & 10.3 \\
\hline Index: & $E_{u}$ & EIP [\%] & EIP [\%] \\
\hline$x m v(1)$ & $2.2 \times 10^{5}$ & 65.8 & -76.1 \\
\hline$x m v(3)$ & $3.9 \times 10^{5}$ & 64.6 & 98.4 \\
\hline$x m v(4)$ & $3.8 \times 10^{4}$ & 41.9 & -2.7 \\
\hline$x m v(5)$ & $9.3 \times 10^{4}$ & 26.9 & 55.0 \\
\hline$x m v(6)$ & $6.6 \times 10^{6}$ & 61.7 & 99.6 \\
\hline$x m v(9)$ & $1.1 \times 10^{4}$ & 80.7 & 37.5 \\
\hline$x m e(21) s p$ & $4.5 \times 10^{5}$ & -46.5 & -144.8 \\
\hline$x m v(11)$ & $1.3 \times 10^{5}$ & 38.6 & 81.4 \\
\hline
\end{tabular}



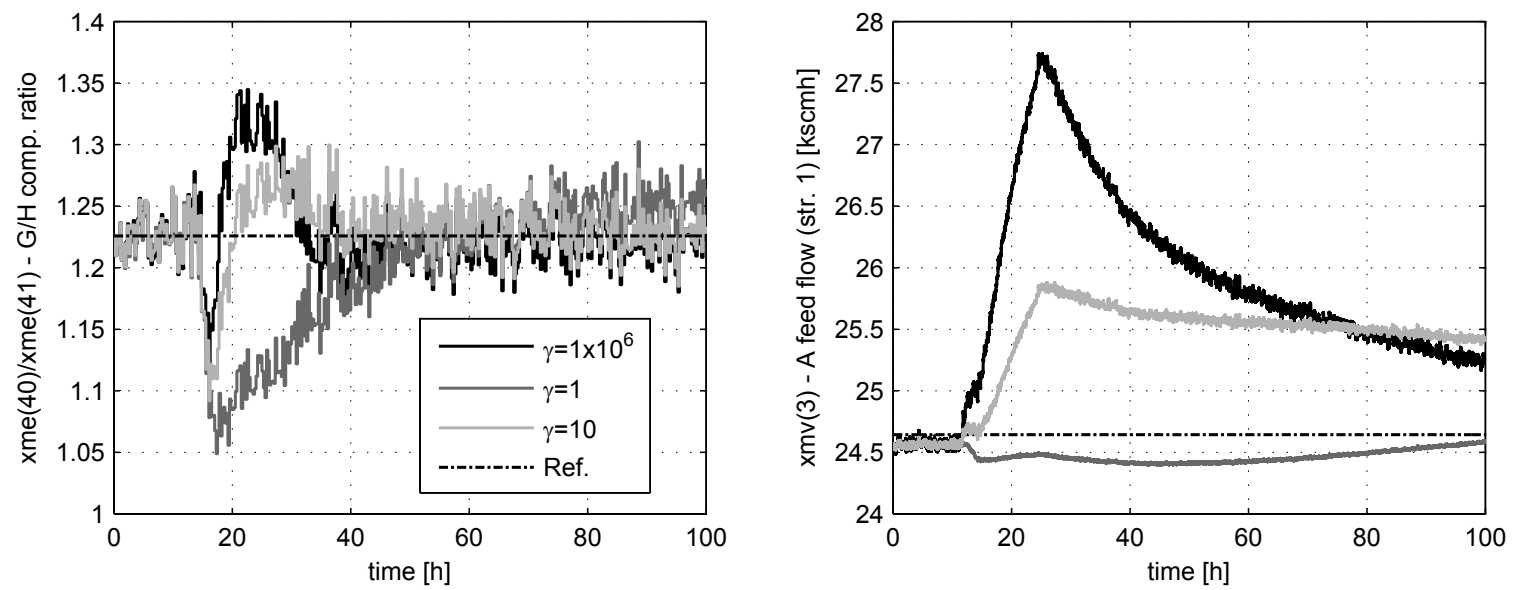

Figure 4: Dynamic responses: (a) $x m e_{G / H}$ and (b) $x m v(3)$
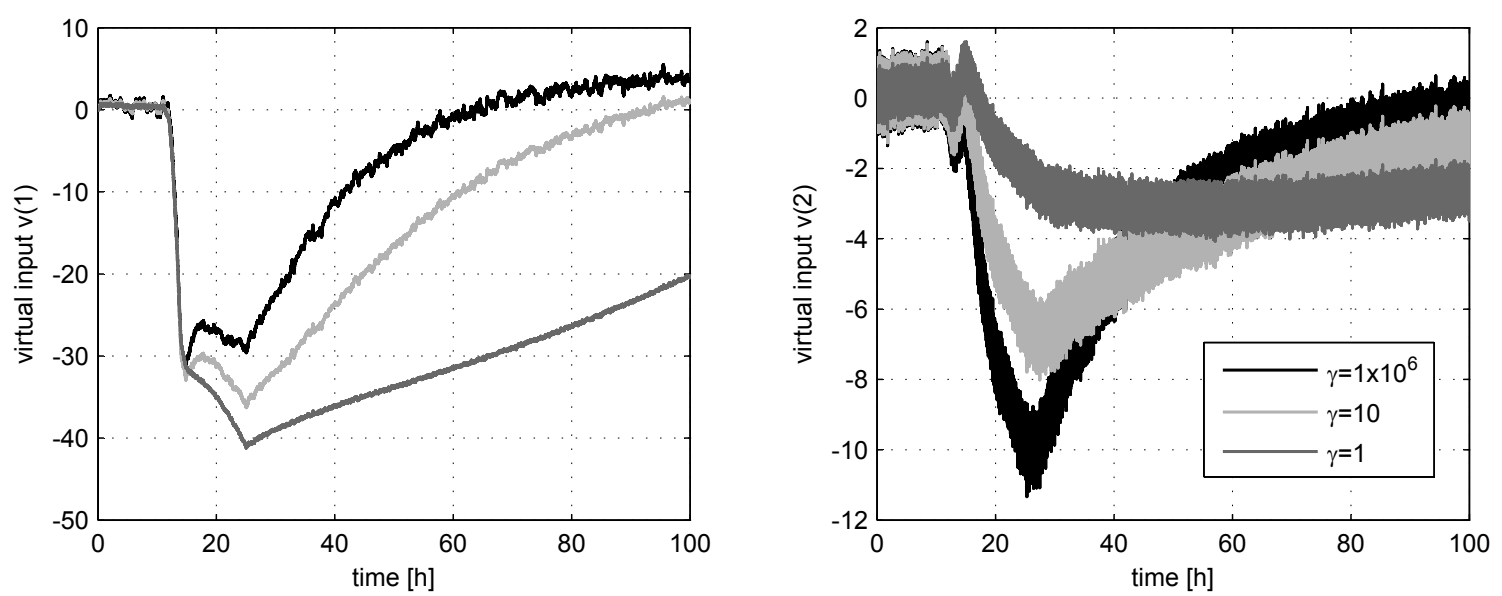

Figure 5: Dynamic responses: (a) $v(1)$ and (b) $v(2)$

(represented by the IAE and $E_{u}$ values, respectively).

As can be seen in Table 7 , for $\gamma=10$ the main outputs reduce their IAE (i.e. they present positive EIP) with respect to the configurations with $\gamma=1 \times 10^{6}$ and $\gamma=1$. Concerning the control energy, a $\gamma=10$ notably improves the performance over the configuration with $\gamma=1 \times 10^{6}$, including the operating costs. Finally, while a $\gamma=1$ further reduces the control energy for certain manipulated variables, the increased error $B u-v$ impacts negatively on the dynamic performance (some outputs present a negative EIP). Hence, $\gamma=10$ is considered here as a reasonable adjustment.

As an example, Fig. 4(a) shows the evolution of the $\mathrm{G} / \mathrm{H}$ comp. ratio $\left(x m e_{G / H}\right)$ for 

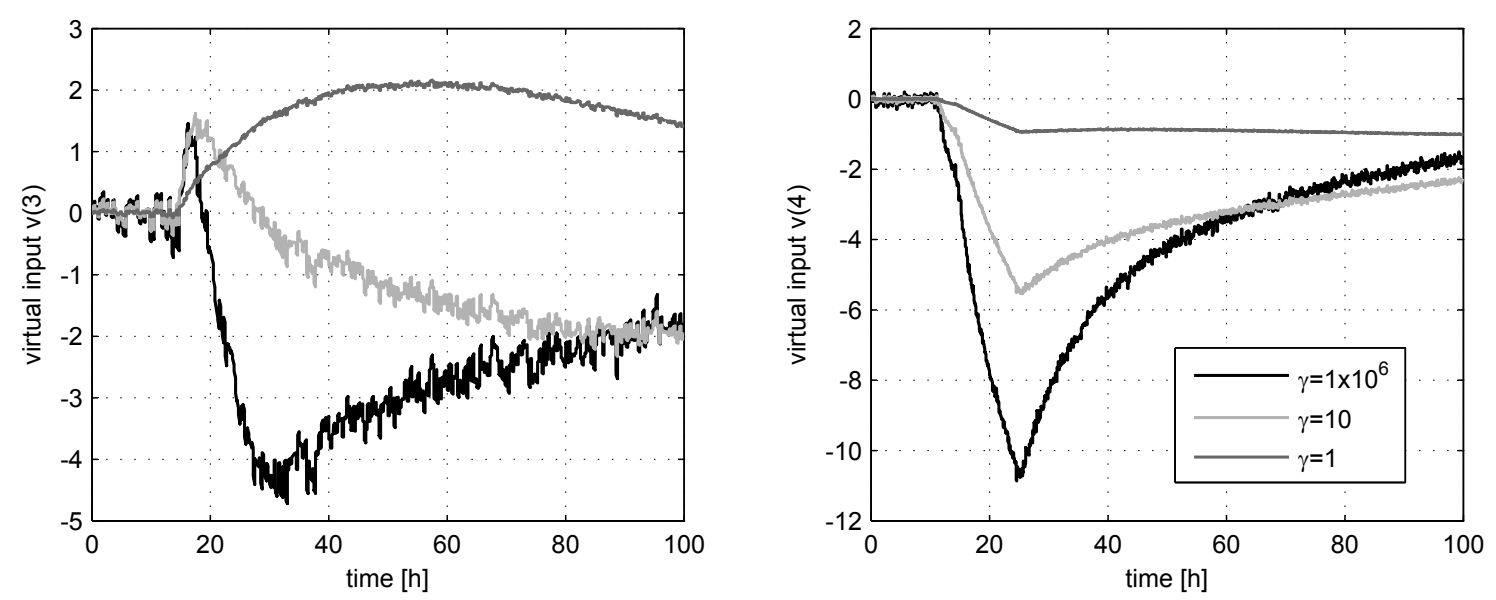

Figure 6: Dynamic responses: (a) $v(3)$ and (b) $v(4)$

alternative $\gamma$ values. The control structure corresponding to $\gamma=10$ presents a very good regulatory behavior subject to disturbances $i d v(1)$ and $i d v(2)$, with a considerable increase in the EIP values with respect to other configurations. In addition, in Fig. 4(b) the A feed flow $(x m v(3))$ response is depicted. As can be seen, the control energy is markedly reduced by implementing the CA block with $\gamma=1$.

Finally, the dynamics of all the virtual variables $v$ are shown in Figs. 5 and 6 . As the $\gamma$ parameter is reduced, the error $B u-v$ increases throughout the time horizon. Note that for $\gamma=1 \times 10^{6}$, this error can be considered null.

The high frequency fluctuations present in several time responses are due to the measurement noise included in the rigorous TE model. As commented in Downs and Vogel, ${ }^{22}$ all process measurements include Gaussian noise with standard deviation typical of the measurement type. 
In the following, it is presented a comparison between: (i) the proposed control structure based on the CA approach (WLS algorithm with $\gamma=10$ ), and (ii) the control strategy presented by Molina et al. ${ }^{23}$ This analysis results interesting given that both proposals utilize all available degrees of freedom (manipulated variables). Table 8 specifies the IAE, EIP and $E_{u}$ indexes related to simulations $\operatorname{Sim}_{5}, \operatorname{Sim}_{6}$ and $\operatorname{Sim}_{7}$. They were computed for key process variables subject to various setpoint changes and disturbance scenarios. Note that a setpoint change corresponding to the reactor pressure $(x m e(7))$ is not included here because this output is not controlled by Molina et al. ${ }^{23}$

First of all, the CA solution as well as the strategy of Molina et al. ${ }^{23}$ meet all control requirements. For all simulation scenarios, the CA strategy presents a very acceptable dynamic behavior where the main outputs have better performance than the solution proposed by Molina et al. ${ }^{23}$ In fact, most EIP indexes result positive. Moreover, the control energy results significantly smaller for most manipulated variables. Note that a fine tuning of the high-level control loops was not performed, nor of the $\gamma$ parameter.

Table 8: Dynamic performance comparison: Molina et al. ${ }^{23}$ solution vs. CA-based strategy

\begin{tabular}{|c|c|c|c|c|}
\hline Index: & EIP [\%] & EIP [\%] & EIP [\%] & $\operatorname{EIP}[\%]$ \\
\hline CA config.: & $\operatorname{WLS}(\gamma=10)$ & WLS $(\gamma=10)$ & WLS $(\gamma=10)$ & WLS $(\gamma=10)$ \\
\hline Sim: & Molina et al. ${ }^{23}$ vs $\operatorname{Sim}_{5}$ & Molina et al. ${ }^{23}$ vs $\operatorname{Sim}_{6}$ & Molina et al. ${ }^{23}$ vs $\mathrm{Sim}_{7}$ & Molina et al. ${ }^{23}$ vs $\mathrm{Sim}_{2}$ \\
\hline Scenario: & $S c_{2}$ & $S c_{4}$ & $S c_{3}$ & $S c_{7}$ \\
\hline$x m e(1)$ & 89.8 & 96.7 & 41.6 & 69.9 \\
\hline$x m e(2)$ & 2.3 & 2.7 & -21.4 & -17.2 \\
\hline$x m e(3)$ & 13.3 & 9.7 & 4.6 & 21.3 \\
\hline$x m e(4)$ & -0.9 & 49.2 & 29.9 & 35.9 \\
\hline$x m e(7)$ & -86.1 & -411.0 & -232.8 & -380.6 \\
\hline$x m e(17)$ & 13.7 & 4.4 & 1.9 & 9.6 \\
\hline$x m e(30)$ & -768.0 & -267.7 & -332.2 & -134.9 \\
\hline$x m e_{G / H}$ & -19.2 & -11.2 & -2.7 & -0.7 \\
\hline Op. costs & -8.2 & 5.4 & -3.6 & 6.1 \\
\hline Index: & EIP $[\%]$ & EIP [\%] & EIP [\%] & EIP [\%] \\
\hline$x m v(1)$ & 4.4 & 5.1 & -131.4 & -88.0 \\
\hline$x m v(3)$ & 99.4 & 99.9 & 73.5 & 98.7 \\
\hline$x m v(4)$ & -0.3 & 75.7 & 75.3 & 80.7 \\
\hline$x m v(5)$ & 96.0 & 99.5 & 84.6 & -83.0 \\
\hline$x m v(6)$ & 89.4 & 89.0 & 86.5 & 94.5 \\
\hline$x m v(9)$ & 99.5 & 99.9 & 99.7 & 99.9 \\
\hline$x m e(21) s p$ & -1076.2 & -57.6 & -1214.6 & -8735.4 \\
\hline$x m v(11)$ & 81.5 & 99.8 & 95.3 & 44.4 \\
\hline
\end{tabular}



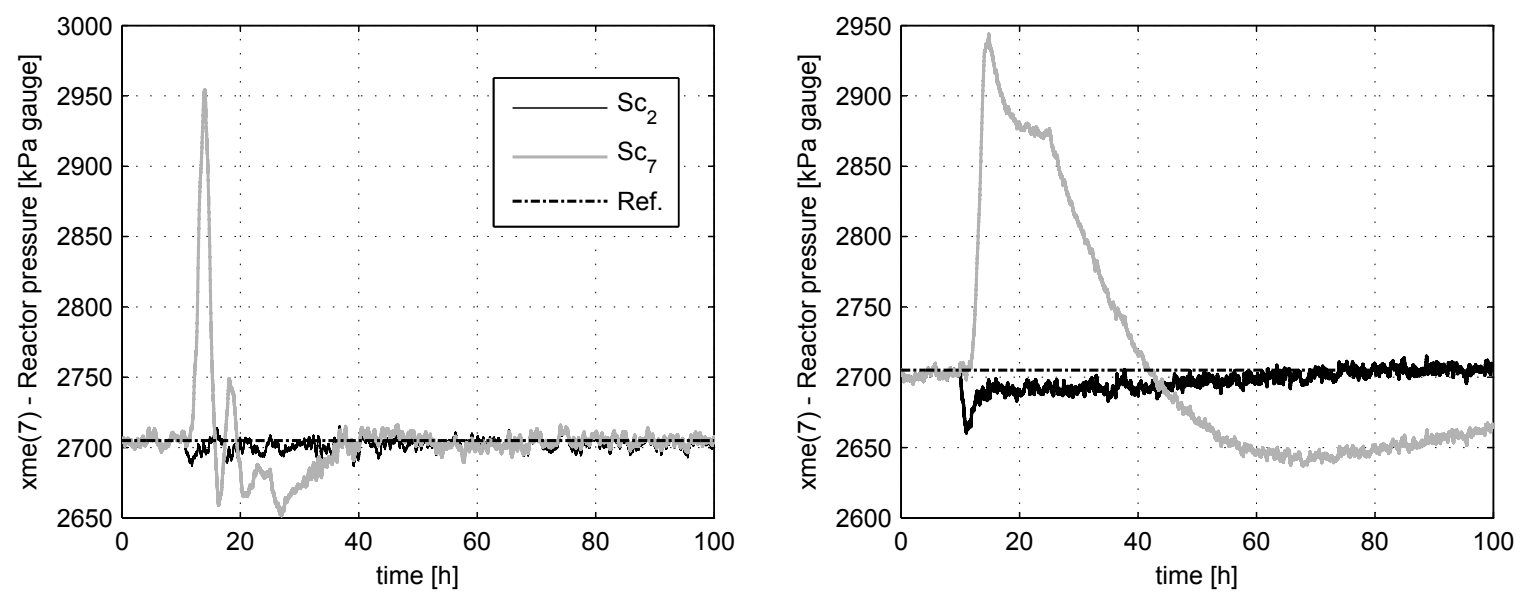

Figure 7: Dynamic responses: (a) $x m e(7)$ (Molina et al. ${ }^{23}$ ) and (b) $x m e(7)$ (CA approach)
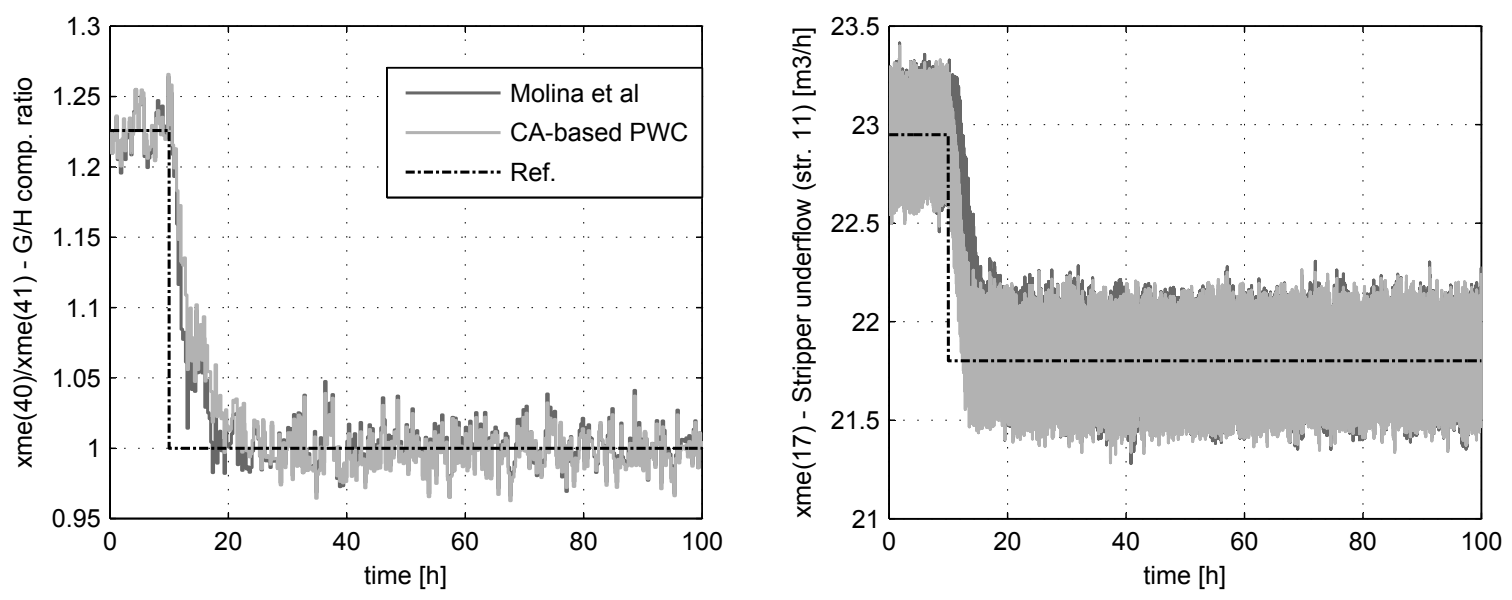

Figure 8: Dynamic responses: (a) $x m e_{G / H}$ and (b) $x m e(17)$

Figs. 7(a) and 7(b) show the evolution of the reactor pressure $(x m e(7))$, obtained respectively with the solution of Molina et al. ${ }^{23}$ and the CA-based strategy. For each case, the regulatory behavior of $x m e(7)$ is presented for two different scenarios: $S c_{2}$ and $S c_{7}$ (see Table 5). As can be seen, the new CA strategy presents acceptable dynamic performance, despite the increased IAE values.

In addition, Figs. 8(a) and 8(b) contrast the servo behavior for both control structures. In Fig. 8(a), a higher IAE value can be noted for the $\mathrm{G} / \mathrm{H}$ comp. ratio $\left(x m e_{G / H}\right)$ with the CA approach. However, the presented servo behavior is very acceptable. In Fig. 8(b), the tracking of the stripper underflow $(x m e(17))$ is depicted. The CA strategy presents good 

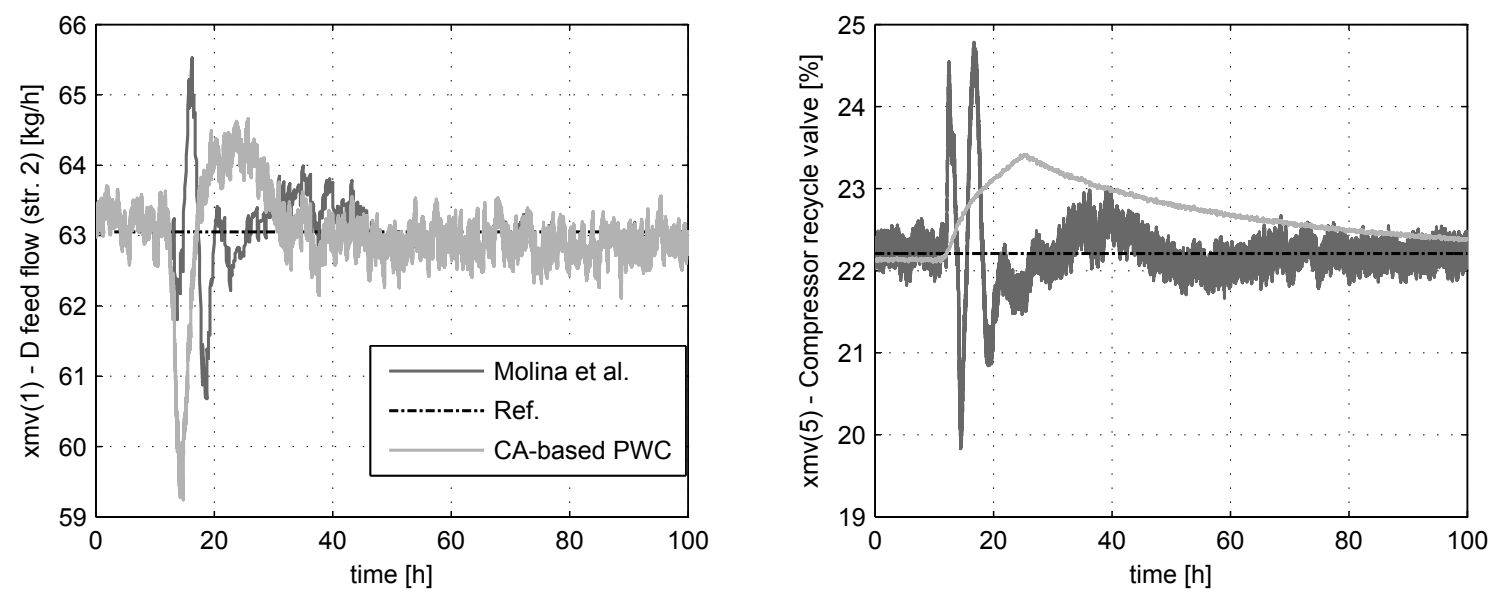

Figure 9: Dynamic responses: (a) $x m v(1)$ and (b) $x m v(5)$

behavior for the setpoint change at $t=10 h$, resulting a reduction of the IAE with respect to the solution proposed by Molina et al. ${ }^{23}$

Figs. 9(a) and 9(b) show the evolution of the D feed flow $(x m v(1))$ and the compressor recycle valve $(x m v(5))$ respectively, subject to the $S c_{7}$ scenario $(i d v(1)$ and $i d v(2))$. While the $E_{u}$ index is increased by the CA strategy, both manipulated variables present a suitable evolution without excessive control energy requirements.

Additionally, in Table S5 (Supporting Information, Appendix E) it is included a comparison between the CA-based structure (WLS with $\gamma=10$ ) and the control strategy developed by Molina et al. ${ }^{23}$ subject to a production rate setpoint change of $+10 \%$. For this simulation, the CA strategy exhibited acceptable dynamic behavior, alternating positive and negative EIP values when compared against the Molina et al. ${ }^{23}$ solution.

Finally, in Table S6 (Supporting Information, Appendix F) it is presented a comparative analysis involving the CA-based structure (WLS with $\gamma=10$ ) and the control strategy proposed by Zumoffen. ${ }^{24}$ The same setpoint changes and disturbance scenarios considered in Table 8 were proposed here. For all simulation scenarios the CA strategy exhibited good dynamic behavior, resulting positive and negative EIP values when compared against the Zumoffen ${ }^{24}$ control structure. While the Zumoffen ${ }^{24}$ strategy employs only 5 of the 8 available manipulated variables, the corresponding control energy is substantially higher. In 
addition, Zumoffen ${ }^{24}$ proposes a decentralized structure which involves 12 PI loops (i.e. $5+$ 7 stabilizing loops). The presented CA-based architecture involves a bi-level structure with 11 PI loops (i.e. $4+7$ stabilizing loops) plus a CA module. However, unlike traditional decentralized structures, the CA approach provides other particular features like constraint handling, management of additional control objectives, etc. as detailed in previous sections. 


\subsubsection{Case 2: severe constraints}

This section starts with a comparison concerning two versions of the CA-based control strategy: (i) Generalized Inverse plus simple saturation, and (ii) WLS approach. For this test, different severe constraint sets presented in Table 6 are considered. The utilized simulation scenarios correspond to $S c_{5}, S c_{6}$ and $S c_{7}$ (see Table 5). In addition, Table 9 shows the IAE, EIP and $E_{u}$ values related to key process variables as suggested by Downs and Vogel. ${ }^{22}$

In general, the WLS algorithm provides better dynamic performance with respect to the generalized inverse method, which does not consider explicitly the constraints. In fact, from Table 9 it can be noted that most outputs reduce their IAE value by implementing the WLS algorithm $\left(\gamma=1 \times 10^{6}\right)$. The disadvantage is the higher control energy requirement, which is reflected by the negative $\operatorname{EIP}\left(E_{u}\right)$ associated with the manipulated variables.

Figs. 10(a) and 10(b) show the evolution of the purge valve $(x m v(6))$ and the A feed flow $(x m v(3))$ respectively, subject to the constraint Set 1 (Table 6) and the $S c_{6}$ scenario (Table $5)$. It is noteworthy that during the activation of the $x m v(6)$ position high limit, the WLS

Table 9: Dynamic performance comparison: Generalized Inverse vs. WLS $\left(\gamma=1 \times 10^{6}\right)$

\begin{tabular}{|c|c|c|c|}
\hline Index: & EIP [\%] & EIP [\%] & EIP [\%] \\
\hline Simulation(s): & $\operatorname{Sim}_{8}$ vs. Sim $_{9}$ & $S_{i m_{10}}$ vs. $S i m_{11}$ & $\operatorname{Sim}_{12}$ vs. $\operatorname{Sim}_{13}$ \\
\hline Constraint set: & Set 1 & Set 2 & Set 3 \\
\hline Scenario: & $S c_{6}$ & $S c_{5}$ & $S c_{7}$ \\
\hline$x m e(1)$ & -6.1 & 0.8 & -28.0 \\
\hline$x m e(2)$ & 4.3 & 7.5 & 23.4 \\
\hline$x m e(3)$ & 0.1 & -15.7 & -6.3 \\
\hline$x m e(4)$ & 2.3 & -3.4 & 8.3 \\
\hline$x m e(7)$ & -0.5 & 0.7 & 9.9 \\
\hline$x m e(17)$ & 3.2 & 10.7 & 19.7 \\
\hline$x m e(30)$ & 0.7 & 5.8 & 1.9 \\
\hline$x m e_{G / H}$ & 3.5 & 13.7 & 38.6 \\
\hline Op. costs & 0.0 & -0.1 & 0.2 \\
\hline Index: & EIP [\%] & EIP [\%] & EIP [\%] \\
\hline$x m v(1)$ & 10.5 & 20.7 & 42.0 \\
\hline$x m v(3)$ & -30.5 & 1.5 & -140.9 \\
\hline$x m v(4)$ & 7.0 & -40.1 & 21.4 \\
\hline$x m v(5)$ & -25.1 & -339.4 & -77.8 \\
\hline$x m v(6)$ & 1.4 & -10.8 & 13.6 \\
\hline$x m v(9)$ & -35.9 & -1398.2 & -426.2 \\
\hline$x m e(21) s p$ & 1.4 & 0.9 & 19.1 \\
\hline$x m v(11)$ & -22.9 & -362.9 & -104.2 \\
\hline
\end{tabular}



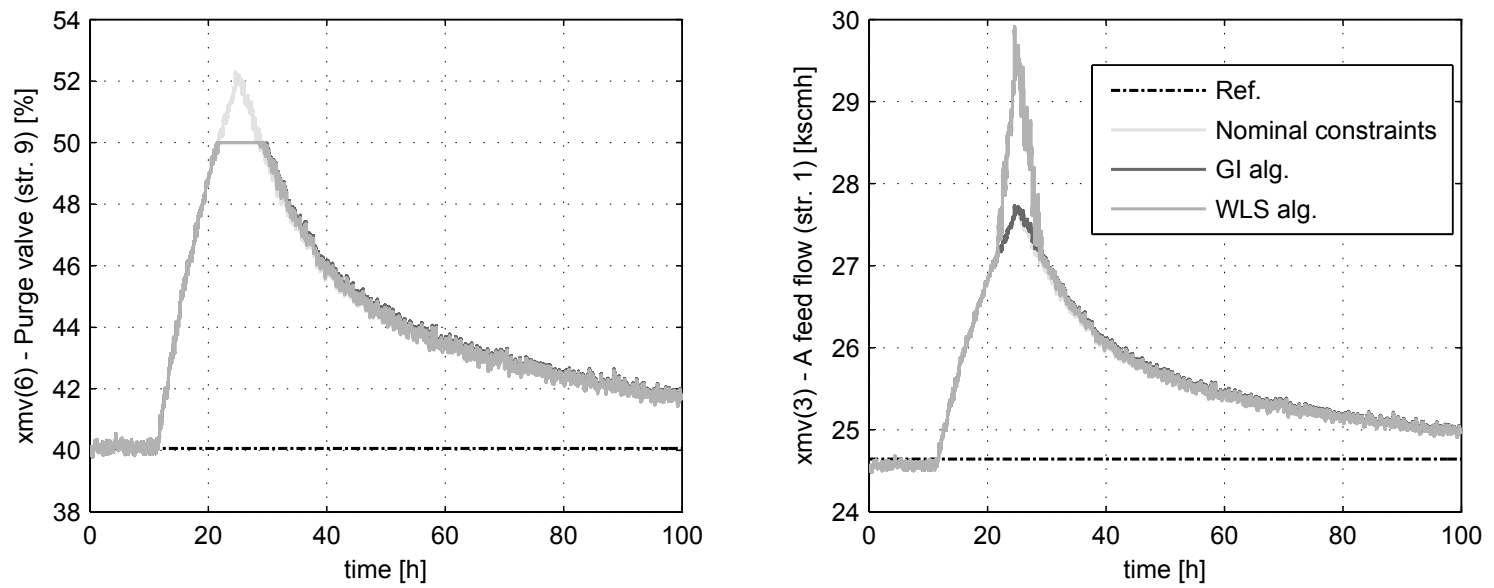

Figure 10: Dynamic responses: (a) $x m v(6)$ and (b) $x m v(3)$
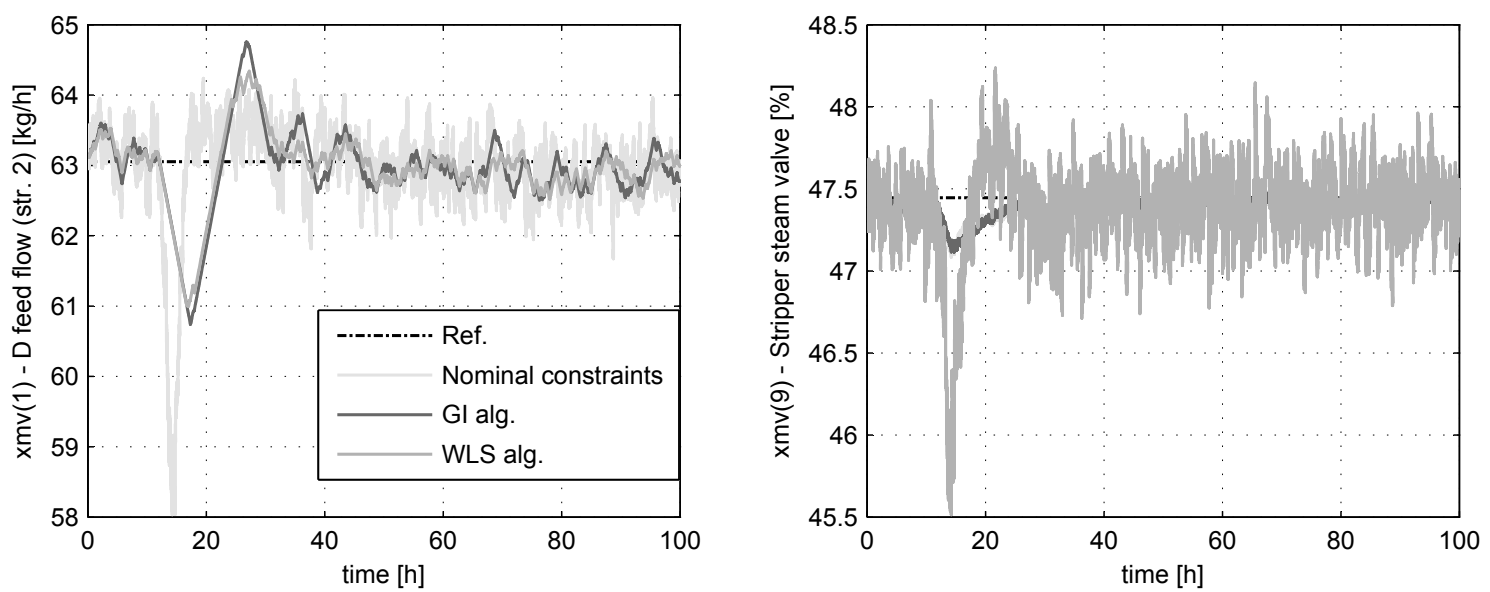

Figure 11: Dynamic responses: (a) $x m v(1)$ and (b) $x m v(9)$

algorithm further manipulates the remaining inputs in order to enhance the system performance. This effect is clearly illustrated in Fig. 10(b), where the control energy requirement is higher $\left(x m v(3)\right.$ has a negative $\left.\operatorname{EIP}\left(E_{u}\right)\right)$.

Figs. 11(a) and 11(b) depict the dynamics of the D feed flow $(x m v(1))$ and the stripper steam valve $(x m v(9))$ respectively, subject to the constraint Set 2 and the $S c_{5}$ scenario. In Fig. 11(a) it can be seen that the constraints become active fewer times with the WLS algorithm. This effect is more evident with the constraints Set 3, which involves position and rate limits, and is analyzed below. Again, the WLS approach utilizes more control energy so as to improve the performance, see Fig. 11(b). 

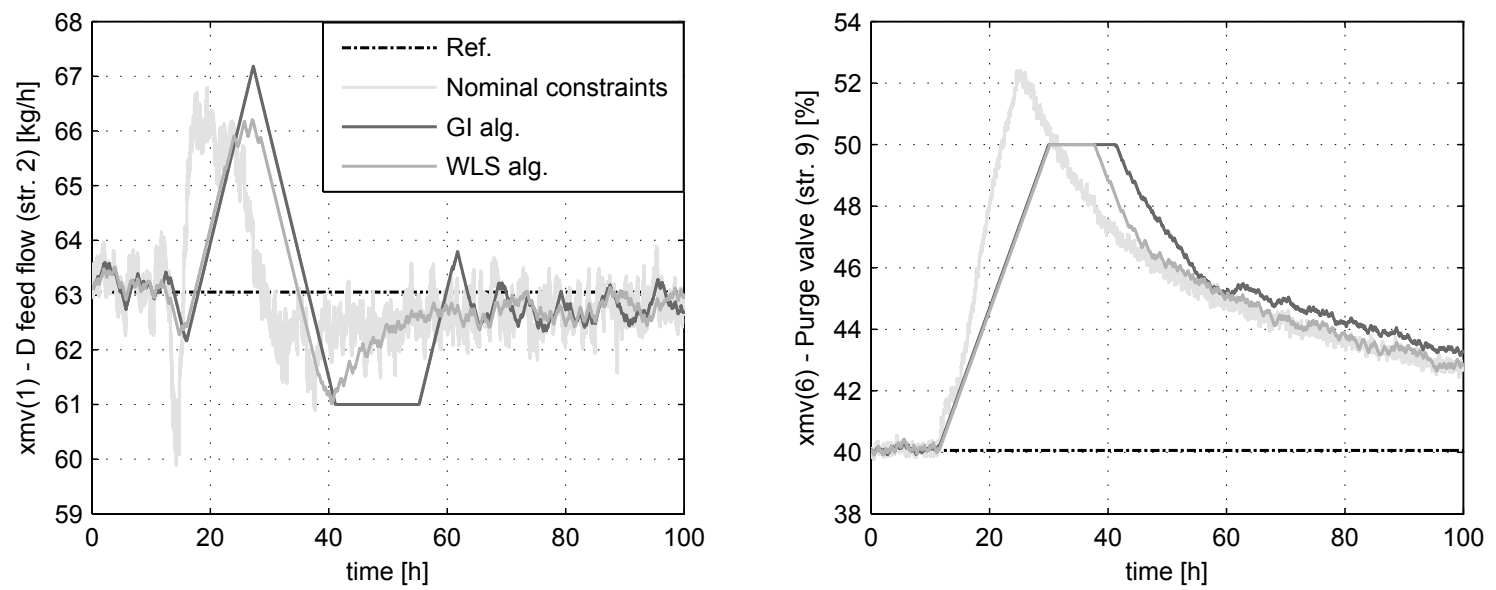

Figure 12: Dynamic responses: (a) $x m v(1)$ and (b) $x m v(6)$
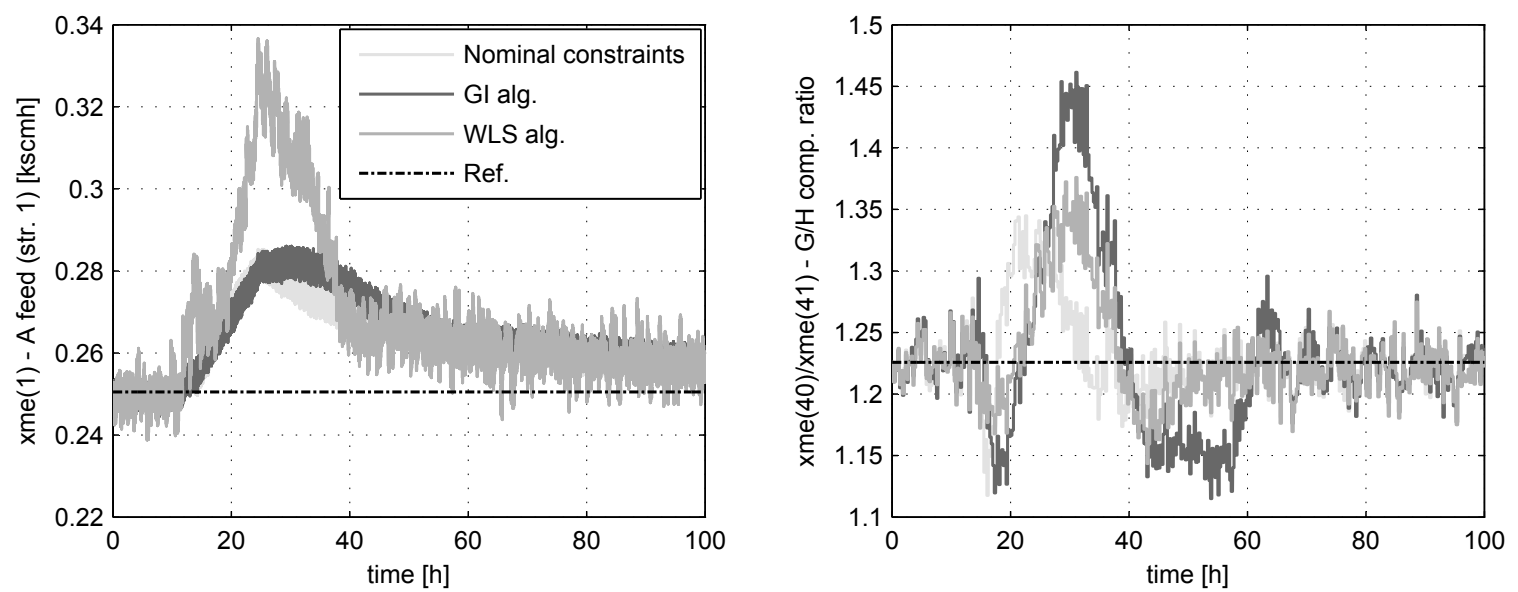

Figure 13: Dynamic responses: (a) $x m e(1)$ and (b) $x m e_{G / H}$

Finally, the evolution of the D feed flow $(x m v(1))$ and the purge valve $(x m v(6))$ subject to the constraints Set 3 and the $S c_{7}$ scenario are presented in Figs. 12(a) y 12(b), respectively. Unlike the WLS, the GI algorithm activates both the position and rate limits for much of the time. As a consequence of this, the corresponding control energy results higher for $x m v(1)$ and $x m v(6)$ manipulated variables.

In Fig. 13(a), the dynamic behavior of the $\mathrm{A}$ feed $(x m e(1))$ is detailed. Despite this output presents a negative EIP, its evolution is acceptable. In addition, Fig. 13(b) shows the $\mathrm{G} / \mathrm{H}$ comp. ratio $\left(x m e_{G / H}\right)$. Its corresponding IAE index is significantly improved by the WLS, which maintains low the variability of this output when compared against the GI 


\subsubsection{Timing analysis}

Here, the timing results corresponding to representative simulations are presented in Table 10. The idea is to evaluate the computational efficiency of the CA block based on the WLS algorithm, which demonstrated very good performance subject to multiple simulation scenarios. In Table 10, WLSC corresponds to a C implementation of the WLS solver. The objective is to analyze the WLS computational requirement in a compiled language. In addition, $C T$ represents the computation time per sample (in milliseconds), and no. iter. corresponds to the number of iterations performed by the WLS per sample. The simulations were performed in Matlab 7.6 running on a $2.8 \mathrm{GHz}$ Dual-Core computer. The tic and toc Matlab commands were used to measure the timing indexes. Note that for the nominal constraints case, the timing properties were averaged over 5 simulations, namely $\mathrm{Sim}_{2}, \mathrm{Sim}_{4}$, $\operatorname{Sim}_{5}, \operatorname{Sim}_{6}$ and $\operatorname{Sim}_{7}$.

As can be seen, the timing measurements are within the same order of magnitude for the different simulations, except for the GI algorithm. In particular, for the WLSC the computational requirement is smaller than for the interpreted Matlab environment. Note that in the worst case, $C T=6.2 \mathrm{~ms}$ per sample. For comparison, the sample time of an industrial DCS/PLC can typically range from a few milliseconds to seconds. In this context, the WLS approach can be considered as an interesting alternative for real-time implementations. Moreover, the WLS allows to limit the number of performed iterations per

Table 10: Timing results

\begin{tabular}{lllllll}
\hline Simulation(s) & Constraints & Algorithm & Max. & Mean & Max. & Mean \\
& & & CT [ms.] & CT [ms.] & no. iter. & no. iter. \\
\hline Sim $_{2,4,5,6,7}$ & \multirow{2}{*}{ Nominal } & WLS & 6.2 & 0.074 & 1 & 1 \\
& & WLSC & 3.3 & 0.042 & 1 & 1 \\
\multirow{4}{*}{$\operatorname{Sim}_{13}$} & GI & 0.5 & 0.005 & - & - \\
& \multirow{2}{*}{ Severe (Set 3) } & WLS & 5.9 & 0.081 & 6 & 1.30 \\
& & WLSC & 3.3 & 0.042 & 6 & 1.30 \\
& & GI & 0.5 & 0.005 & - & - \\
\hline
\end{tabular}


sample. This is advantageous for scheduling the WLS routine in a DCS/PLC application. However, for the case that the WLS needs more iterations than those stipulated, it will produce a suboptimal solution and probably a degradation of the system performance.

\section{Conclusions}

The proposed plantwide control methodology combines the design of a decentralized control structure with the configuration of a control allocation module. While the decentralized structure can be synthesized with classic PI controllers, there are different alternatives for the CA module. In this work, the weighted least-squares (WLS) formulation based on an active set method promoted by Harkegard ${ }^{21}$ was adopted due to the obtained trade-off between dynamic quality and computational load. The suggested mixed structure offers several valuable features that are usually provided by MPC-based strategies (e.g. constraint handling and management of secondary control objectives) but without requiring complex process modeling or high computation times. In fact, the complete control structure can be designed taking into account a steady-state model of the process. The operating philosophy is relatively simple to understand and it requires the adjustment of a few parameters for its basic operation. The obtained simulation times show that the proposed CA-based strategy can be considered as an interesting alternative for real-time industrial implementations.

In this study the efficiency of the genetic algorithm (GA) was not prioritized since it is executed few times, in offline mode. The GA average computation time resulted 1104.5 seconds on a desktop computer with Intel Core i7 (3.40 GHz, 12 Gb RAM) using Matlab r2014a. Recently, the author's working group published a new approach to address multivariable control structure design based on a mixed-integer quadratic programming model. ${ }^{36}$ In contrast to the GA method, this new contribution guarantees the optimality of the solution and significantly improves the computation times. In future CA-based designs, the methodology presented in Braccia et al. ${ }^{36}$ will be employed in order to obtain the high-level 
control structure.

Actually, an advanced version of the CA-based methodology is being evaluated in order to obtain fault-tolerant control structures for the bio-ethanol fuel processor system (BPS) with fuel cell (FC). ${ }^{15}$ This involves on the one hand, the modeling of the faults to be dealt with, and on the other hand, the appropriate (online) adaptation of the CA module. The objective of the fault-tolerant strategy will be to properly distribute the primary control actions along the healthy actuators, avoiding the reconfiguration of the high-level controller.

\section{Acknowledgement}

The authors thank the financial supports from CONICET (Consejo Nacional de Investigaciones Científicas y Técnicas) and UNR-FCEIA (Universidad Nacional de Rosario). The authors also acknowledge the support from UTN-FRRo (Universidad Tecnológica Nacional) and CIFASIS (Centro Internacional Franco Argentino de Ciencias de la Información y de Sistemas).

\section{Supporting Information Available}

Main parameter settings, implementation details and dynamic performance evaluation of the proposed control structures. This material is available free of charge via the Internet at http://pubs.acs.org/. 


\section{References}

(1) Downs, J.; Skogestad, S. An industrial and academic perspective on plantwide control. Annu. Rev. Control 2011, 35, 99-110.

(2) Correa de Godoy, R.; Garcia, C. Plantwide Control: A Review of Design Techniques, Benchmarks, and Challenges. Ind. Eng. Chem. Res. 2017, DOI: 10.1021/acs.iecr.7b00416.

(3) Rangaiah, G. P.; Kariwala, V. Plantwide Control. Recent Developments and Applications; John Wiley \& Sons, 2012.

(4) Campo, P.; Morari, M. Achievable closed-loop properties of systems under decentralized control: conditions involving the steady-state gain. IEEE Transactions on Automatic Control 1994, 39, 932-943.

(5) Luppi, P.; Basualdo, M. Smart Investment for Redundancies Selection Integrated to Reconfigurable Fault-Tolerant Control Design. Ind. Eng. Chem. Res. 2016, 55, 94859497.

(6) Stephanopoulos, G.; Reklaitis, G. Process systems engineering: From Solvay to modern bio- and nanotechnology. A history of development, successes and prospects for the future. Chem. Eng. Sci. 2011, 66, 4272-4306.

(7) Sharifzadeh, M. Integration of process design and control: A review. Chem. Eng. Res. Des. 2013, 91, 2515-2549.

(8) Huang, R.; Liu, Y.; Zhu, J. Guidance, navigation and control system design for tripropeller vertical-takeoff-and-landing unmanned air vehicle. J. Aircraft 2009, 46, 18371856.

(9) Feemster, M.; Esposito, J. Comprehensive framework for tracking control and thrust 
allocation for a highly overactuated autonomous surface vessel. J. Field Robot 2011, 28, 80-100.

(10) Mokhiamar, O.; Abe, M. How the four wheels should share forces in an optimum cooperative chassis control. Control Eng. Pract. 2006, 14, 295-304.

(11) Johansen, T.; Fossen, T. Control allocation - A survey. Automatica 2013, 49, 10871103.

(12) Harkegard, O.; Glad, T. Resolving actuator redundancy. Optimal control vs. control allocation. Automatica 2005, 41, 137-144.

(13) Alwi, H.; Edwards, C. Fault tolerant control using sliding modes with online control allocation. Automatica 2008, 44, 1859-1866.

(14) Luppi, P.; Outbib, R.; Basualdo, M. Nominal Controller Design Based on Decentralized Integral Controllability in the Framework of Reconfigurable Fault-Tolerant Structures. Ind. Eng. Chem. Res. 2015, 54, 1301-1312.

(15) Luppi, P.; Nieto Degliuomini, L.; Garcia, M.; Basualdo, M. Fault-tolerant control design for safe production of hydrogen from bio-ethanol. Int. J. Hydrogen Energy 2014, 39, $231-248$.

(16) Casavola, A.; Garone, E. Fault-tolerant adaptive control allocation schemes for over actuated systems. Int J. Robust Nonlin 2010, 20, 1958-1980.

(17) Skogestad, S.; Postlethwaite, I. Multivariable feedback control. Analysis and design; John Wiley \& Sons, 2005.

(18) Zumoffen, D. Plant-wide control design based on steady-state combined indexes. ISA T. 2016, 60, 191-205.

(19) Petersen, J.; Bodson, M. Constrained quadratic programming techniques for control allocation. IEEE T. Contr. Syst. T. 2006, 14, 91-98. 
(20) Bodson, M. Evaluation of Optimization Methods for control allocation. J. Guid. Control Dynam. 2002, 25, 703-711.

(21) Harkegard, O. Efficient active set algorithms for solving constrained least squares problems in aircraft control allocation. Proceedings of the 41 st IEEE Conference on Decision and Control 2002,

(22) Downs, J. J.; Vogel, E. F. A plant-wide industrial process control problem. Comput. Chem. Eng. 1993, 17, 245-255.

(23) Molina, G.; Zumoffen, D.; Basualdo, M. Plant-wide Control Strategy Applied to the Tennessee Eastman Process at Two Operating Points. Comput. Chem. Eng. 2011, 35, $2081-2097$.

(24) Zumoffen, D. Oversizing analysis in plant-wide control design for industrial processes. Comput. Chem. Eng. 2013, 59, 145-155.

(25) Rivera, D. Una metodologia para la identificacion integrada con el diseno de controladores IMC-PID. Revista Iberoamericana de Automatica e Informatica Industrial 2007, 4, 5-18.

(26) Garcia, C.; Morari, M. Internal model control. 2. Design procedure for multivariable systems. Industrial and Engineering Chemistry Product Research and Development 1985, 24, 472-484.

(27) Leskovec, J.; Rajaraman, A.; Ullman, J. Mining of Massive Datasets; Cambridge University Press, 2014.

(28) Klema, V.; Laub, A. The Singular Value Decomposition: Its Computation and Some Applications. IEEE T. Automat. Contr. 1980, 2, 164-176.

(29) Kariwala, V.; Cao, Y. Branch and Bound method for multiobjective pairing selection. Automatica 2010, 46, 932-936. 
(30) McAvoy, T. A methodology for screening level control structures in plantwide control systems. Comput. Chem. Eng. 1998, 22, 1543-1552.

(31) Arkun, Y.; Downs, J. A general method to calculate input-output gains and the relative gain array for integrating processes. Comput. Chem. Eng. 1990, 14, 1101-1110.

(32) Branke, J.; Deb, K.; Miettinen, K.; Slowinski, R. Multiobjective Optimization. Interactive and Evolutionary Approaches; Springer, 2008.

(33) Wang, Z.; Rangaiah, G. Application and Analysis of Methods for Selecting an Optimal Solution from the Pareto-Optimal Front obtained by Multiobjective Optimization. Ind. Eng. Chem. Res. 2017, 56, 560-574.

(34) Luppi, P.; Zumoffen, D.; Basualdo, M. Decentralized plantwide control strategy for large-scale processes. Case study: Pulp mill benchmark problem. Comput. Chem. Eng. 2013, 52, 272-285.

(35) Konda, N.; Rangaiah, G. Performance assessment of plantwide control systems of industrial processes. Ind. Eng. Chem. Res. 2007, 46, 1220-1231.

(36) Braccia, L.; Marchetti, P.; Luppi, P.; Zumoffen, D. Multivariable Control Structure Design Based on Mixed-Integer Quadratic Programming. Ind. Eng. Chem. Res. 2017, $56,11228-11244$. 


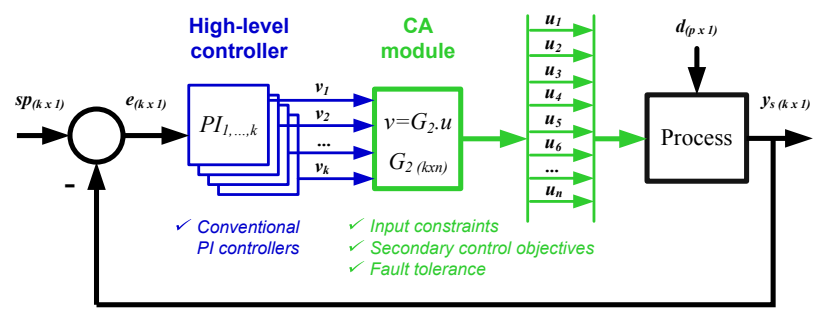

Figure 14: Abstract Graphic 


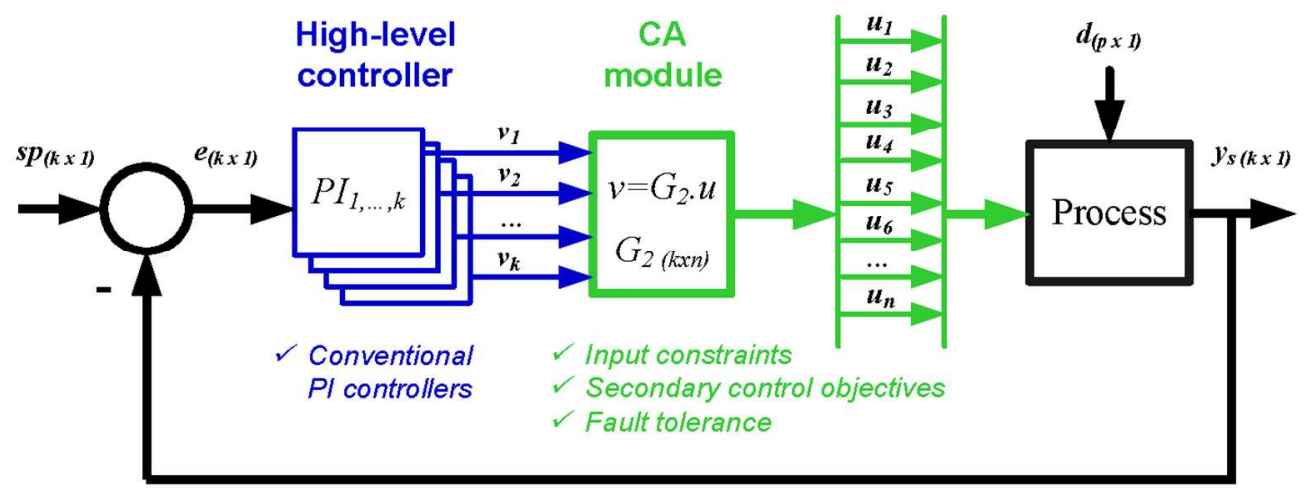

$122 \times 46 \mathrm{~mm}(300 \times 300$ DPI $)$ 